\title{
ANÁLISIS COMPARATIVO DE LA EFICACIA PUBLICITARIA EN TELEVISIÓN: TELEPROMOCIÓN VERSUS SPOT*
}

\section{COMPARATIVE ANALYSIS OF EFFICIENCY IN TELEVISION ADVERTISING: TELEPROMOTION VERSUS SPOT}

\author{
Josefa D. Martín Santana *1 \\ UNIVERSIDAD DE LAS PALMAS DE GRAN CANARIA \\ Eva M. Reinares Lara ${ }^{2}$ y Pedro J. Reinares Lara $^{3}$
UNIVERSIDAD REY JUAN CARLOS
}

\section{RESUMEN}

La publicidad en televisión se está desarrollando más allá de los cortes publicitarios tradicionales. Esta tendencia requiere considerar cómo la publicidad y el contenido de la programación televisiva pueden utilizarse conjuntamente con fines comerciales. En este trabajo se presentan los resultados de una investigación que analiza, en un entorno real, los efectos cognitivos, afectivos y conativos de un formato de publicidad no convencional, la Telepromoción externa, frente a la publicidad más convencional, el Spot, sobre los diferentes per- files sociodemográficos de las audiencias de televisión. También se plantea el análisis de una serie de variables relativas al individuo que potencialmente pueden ejercer alguna influencia sobre tales efectos. De esta manera se han podido aportar las necesarias evidencias empíricas para el desarrollo teórico de las formas no convencionales de publicidad en televisión, y justificar adecuadamente su aplicación en la práctica empresarial.

Palabras clave: Publicidad en televisión, eficacia publicitaria, nuevas formas de publicidad o formatos publicitarios no convencionales.

\footnotetext{
* Esta investigación ha sido financiada con el Proyecto de Investigación del "Programa para la Creación y Consolidación de Grupos de Investigación" (BOCM 11 de julio de 2006) de la Comunidad de Madrid y la Universidad Rey Juan Carlos.

** Los autores agradecen los comentarios y sugerencias propuestas por los evaluadores anónimos a una primera versión de este trabajo.

1 Profesora Titular de Universidad. Universidad de Las Palmas de Gran Canaria. Facultad de Ciencias Económicas y Empresariales. Departamento de Economía y Dirección de Empresas. Edificio Empresariales. Módulo C. Campus de Tafira. 35017. Islas Canarias. E-mail.jmartin@dede.ulpgc.es

2 Profesora Titular de Universidad. Universidad Rey Juan Carlos. Facultad de Ciencias Jurídicas y Sociales. Departamento de Economía de la Empresa. Paseo de los Artilleros s/n. Campus de Vicalvaro. 28032. Madrid. E-mail. eva.reinares@urjc.es.

3 Profesor Titular de Universidad. Universidad Rey Juan Carlos. Facultad de Ciencias Jurídicas y Sociales. Departamento de Economía de la Empresa. Paseo de los Artilleros s/n. Campus de Vicalvaro. 28032. Madrid. E-mail. pedro.reinares@urjc.es.
} 


\section{ABSTRACT}

Television advertising is moving beyond traditional commercial breaks. This tendency requires to consider how advertising and television programming content can be used together in order to set commercial goals. This work presents the results from a research that analyzes, in a real environment, the cognitive, affective and conative effects of a non-conventional advertising format, the external telepromotion, as opposite to more conventional advertising, the spot. These results are presented differentiating between the different sociodemographic profiles of the television audiences. Also the analysis of a series of variables related to the individual that can potentially exercise some influence over such effects are showed. This way, enough empirical evidence for theorical development on non-conventional advertising on television has been provided, and so, arguments to justify its application in business practice.

Keywords: Television advertising, advertising effectiveness, new advertising forms or non-conventional advertising format.

\section{INTRODUCCIÓN}

Desde hace ya una década, la eficacia publicitaria de los anuncios convencionales de televisión se ha cuestionado tanto en la praxis empresarial como en el ámbito académico (DAWSON, 1996; DAVIES Y ROJAS-MÉNDEZ, 2005; Mediaedge, 2007; Wilbur, 2008). Las razones hacen referencia principalmente a la fragmentación de la audiencia (CHOWDHURY, ET $A L .$, 2007; SHARP ET AL., 2009), el zapping (Heeter Y Greemberg, 1985; Tse Y Lee, 2001; Elpers ET AL., 2003; GÁzQuez ET $A L$., 2010) y la saturación (REINARES Y REINARES, 2007; SOFRES, 2008). Estos obstáculos exigen de un mayor uso creativo del medio por parte de anunciantes, cadenas de televisión, agencias de publicidad y agencias de medios, con el objetivo general de conseguir mayor eficacia en las campañas (D’Astous y SÉGuin, 1999; JAFFe, 2005; SMIT ET AL., 2009).

En este contexto, la publicidad televisiva se está desarrollando más allá de los cortes publicitarios tradicionales (EGTA, 2008a; FARRÉ Y FERNÁNDEZ, 2005). Este hecho requiere replantarse cómo la publicidad y el contenido de la programación televisiva se pueden utilizar conjuntamente con fines comerciales (HACKLEY Y TIWSAKUL, 2006).

No existe consenso para denominar a estas nuevas formas de utilización comercial del medio televisión. Tanto en el sector empresarial como en el académico se utilizan diversos términos para referirse a este conjunto de formatos publicitarios tales como "Publicidad especial", "Nuevos formatos de publicidad en televisión" o "Brand placement" (SMIT ET AL., 2009). La legislación de rango europeo regula de forma especifica estas formas de publicidad bajo la denominación de "Otras formas de publicidad" y "Nuevas formas de publicidad" (Articulo 18 (1), Audio Visual Media Services without Frontiers (AVMA) Directive ${ }^{4}$ ) (EUROPEAN COMMISSION, 2007). Estos términos integran todas las formas de publicidad en televisión diferentes explícitamente de los Spots (publicidad convencional) con sus distintas duraciones (WoODs, 2008). También haciendo referencia, por oposición, a toda comunicación comercial que no se presente en forma de anuncios, FARRÉ Y FERNÁNDEZ (2005) proponen como la mejor denominación posible "Publicidad no convencional". Adhiriéndonos a esta línea de nomenclatura, en el presente trabajo se utiliza el término "Formas No Convencionales" (FNC) para referirnos a este conjunto de formatos publicitarios en el medio televisión.

Con la creciente importancia de las alternativas al Spot de 20 segundos y la progresiva gama de oportunidades que brindan los nuevos cana-

\footnotetext{
${ }^{4}$ Directiva 2007/65/CE del Parlamento Europeo y del Consejo, de 11 de diciembre de 2007.
} 
les de televisión, el medio ofrece un amplio catálogo de nuevas formas de hacer publicidad con características, gestión y objetivos de comunicación diferentes del Spot. Se han encontrado una multiplicidad de nuevos forma- tos de publicidad en las ofertas comerciales presentadas por las televisiones en los diferentes países examinados (CARAT CRISTAL Y BIRD \& BIRD, 2002). El Cuadro 1 presenta una clasificación de los diferentes formatos televisivos.

CUADRO 1

Clasificación de los formatos publicitarios en televisión en función de su asociación con los programas

\begin{tabular}{|l|l|}
\hline NO ASOCIADAS A LOS PROGRAMAS & ASOCIADAS A LOS PROGRAMAS \\
\hline Spots & Product placement \\
\hline $\begin{array}{l}\text { Microprogramas con contenido publicitario } \\
\text { (Publireportajes) y sin contenido publicitario }\end{array}$ & $\begin{array}{l}\text { Caretas de patrocinio, de avance de patrocinio y de } \\
\text { sección. }\end{array}$ \\
\hline $\begin{array}{l}\text { Cortinillas de integración con la comunicación } \\
\text { corporativa de la cadena }\end{array}$ & Telepromociones internas y externas \\
\hline Teletienda & Sobreimpresiones \\
\hline Infocomercials & Pantalla compartida \\
\hline
\end{tabular}

Existen muchas evidencias sobre la creciente popularidad de otras formas de publicidad televisiva (LOWREY ET AL., 2005; VON RIMSCHA, ET AL., 2008; RuBInSON, 2009). Atendiendo a criterios exclusivamente cuantitativos, representan entre el $5 \%$ y el $15 \%$ de los ingresos publicitarios de los organismos de radiodifusión (EGTA, 2008b). En España, por ejemplo, el conjunto de las televisiones han facturado en 2008 por estos formatos más de 325 millones de euros, el $10 \%$ de sus ingresos publicitarios; multiplicando por 3,6 los apenas 90 millones de euros que suponían en 2000 (ARRAzola et. al., 2010). Incluso en el ámbito regulador europeo (Directiva 2007/65/CE), que tradicionalmente va unos pasos detrás de la evolución de los usos y costumbres en la comunicación, se reconoce la importancia de estos nuevos formatos como medio para dar mayor flexibilidad a la financiación de los contenidos audiovisuales (REINARES et. al., 2007).

Este escenario proporciona el impulso para la investigación en la planificación y ejecución de estas formas de publicidad televisiva, en la medida en la que la consolidación de estos formatos recomienda contrastar empíricamente y con rigor su eficacia frente a la publicidad convencional.

La revisión de la literatura académica de referencia presenta ciertas limitaciones a la hora de proporcionar una explicación científica e integral del fenómeno. Como dificultades concretas se pueden destacar las siguientes:

- La mayoría de las investigaciones aplicadas al estudio de la eficacia publicitaria en televisión se centran en los Spots, cuyas características formales hacen que las implicaciones de dichos trabajos, en algunos casos, tengan limitada consideración como antecedente teórico.

- Existe un limitado número de investigaciones que conceptualicen adecuadamente las distintas tipologías de FNC. Esto impide caracterizar formatos que son profusamente utilizados en las prácticas publicitarias (ARRAZOLA ET AL., 2010). 
- Los trabajos consultados aportan una descripción parcial del fenómeno que dificulta el desarrollo de un marco teórico relevante.

- Los resultados obtenidos en dichos trabajos son contradictorios o escasamente concluyentes, por sus objetivos exploratorios o su carácter experimental. Los propios autores reconocen las limitaciones de sus aportaciones y proponen como futura línea de investigación aplicar otras metodologías (VAKRATSAS, 2005).

Lo anteriormente expuesto ha permitido identificar una parcela inexplorada que constituye el objeto de investigación del presente trabajo de investigación, con el que se pretende contribuir al estudio de los efectos de las 1lamadas formas no convencionales de publicidad en televisión al superar algunas de las limitaciones identificadas.

DAHLÉN Y EDENIUS (2007) proponen que las formas publicitarias no convencionales en televisión deben ser estudiadas a partir de objetivos similares a los propios de la publicidad convencional. En este contexto, los objetivos de este trabajo desarrollan la secuencia habitual en los procedimientos de medición de eficacia publicitaria, que los clasifica en relación con las etapas conocer-sentir-hacer de los modelos de jerarquía de efectos de la publicidad.

Del conjunto de formas publicitarias no convencionales se ha elegido para la investigación comparativa con el Spot, la "Telepromoción externa". La elección de esta forma publicitaria se debe a su uso consolidado y creciente por parte de los anunciantes y su comercialización en la mayor parte de cadenas generalistas europeas (EGTA, 2008b). Las Telepromociones externas son una FNC en la que personas y personajes de una cadena, sirviéndose de escenarios y/o situaciones notorias de la ficción de la cadena, realizan la comunicación comercial del anunciante a partir de un desarrollo creativo basado en teleseries $u$ otras situaciones inherentes al papel habitual de cada rostro conoci- do en la cadena (REINARES Y REINARES, 2003; 223). Para reforzar la asociación suelen emitirse antes o durante la serie de la que se sirven para crear complicidad entre su trama y la publicidad.

Así, la presente investigación plantea analizar, con el adecuado rigor científico en un entorno real, los efectos cognitivos, afectivos y de conducta de un formato de publicidad no convencional, la Telepromoción externa, frente al Spot, sobre los diferentes perfiles sociodemográficos de las audiencias de televisión. Con el mismo criterio se plantea el análisis de una serie de variables relativas al individuo que pueden ejercer alguna influencia sobre tales efectos.

\section{FORMATOS NO CONVENCIONALES DE PUBLICIDAD EN TELEVISIÓN}

BALASUBRAMANIAN (1994) definió estas formas de publicidad como acciones con un pago al medio con el objetivo de influir en un público para obtener beneficios comerciales por medio de comunicaciones que proyectan un carácter no comercial. Este hecho dificulta en los espectadores la distinción entre los mensajes promocionales y de entretenimiento. El autor acuña el término "mensajes híbridos", para caracterizar esta comunicación comercial que mezcla publicidad (persuasión) e información.

HACKLEY Y TIWSAKUL (2006), tras observar la falta de consenso en la investigación académica respecto a la conceptualización de estas formas de comunicación persuasiva, introducen el término "Entertainment marketing", que integra cualquier forma de presentar la marca dentro de contenido propio del medio televisivo. La práctica del Entertainment marketing crea una relación simbiótica entre la comunicación persuasiva y los programas. Esta conceptualización de las FNC a partir de la integración de los mensajes comerciales con los contenidos televisivos se recoge en fuentes posteriores (SMIT ET AL., 2009). 
Dada la confusión que se produce en la literatura al describir las diferentes FNC es importante conceptualizar adecuadamente las mismas. Las FNC son mensajes híbridos (publicidad más entretenimiento), a pesar de que la ley que les afecta las reconoce y define como publicidad (FARRÉ y FERNÁNDEZ, 2005). Por lo tanto, se trata de mensajes publicitarios explícitos, que no esconden sus objetivos persuasivos, pero sí se benefician de diferentes niveles de integración con los soportes televisivos y sus programas. Sin embargo, las FNC tienen características formales que las diferencian del formato publicitario denominado Spot (publicidad convencional en televisión). El Spot se integra en los espacios publicitarios de forma independiente, mientras que las FNC aportan un valor añadido en su capacidad de persuasión, integrándose de diferentes formas ${ }^{5}$ junto a los programas y desarrollando su creatividad aprovechando cualidades del espacio que le antecede o precede.

Las principales características que diferencian las FNC de los Spots se citan a continuación:

- Son formas de comunicación fuera del bloque publicitario (dentro de los programas) o anexadas al principio o fin de los programas.

- Están integradas en diferentes niveles dentro de los contenidos de los programas; por ejemplo, mediante su involucración en los magazines a través de sus presentadores y escenarios, o en las series de ficción a través de los personajes interpretados por sus actores y los distintos escenarios donde se graban.

- Buscan la asociación con los programas y sus contenidos; o basando su creatividad en los contenidos de los programas afines a las audiencias que interesan comercialmente.
- Sus contenidos son específicos para cada cadena y programa: no se repiten y no se intercambian entre las cadenas. Este es uno de los elementos que más explícitamente diferencia los Spots de las FNC; los Spots se emiten de forma idéntica en todas las cadenas elegidas por el planificador y son independientes de éstas y sus contenidos, mientras que cada FNC es específica de la cadena que la emite y de sus contenidos propios.

- Su gestión no es exclusiva de los departamentos comerciales de las cadenas. En ella pueden intervenir productoras, agencias y centrales.

- Están sometidas a un régimen legal específico (Ej.: tiempos máximos por hora) que las diferencia de los Spots (EUROPEAN Comision, 2007; OfCom, 2005). Dado que a veces pueden ser confundidas por las audiencias con los contenidos no comerciales, la ley obliga a su identificación expresa como publicidad (LEY GENERAL DE LA COMUNiCACiÓN AUdiOvisual, 2010).

El grueso de la literatura sobre FNC se centra, por un lado, en la caracterización de la naturaleza de las FNC (conceptualización, diferencias con respecto a otras formas de publicidad y comunicación, y análisis descriptivo de los elementos formales que permiten clasificar las diferentes tipologías de formas no convencionales) y, por otro, en las implicaciones prácticas y aspectos relacionados directamente con la gestión en su sentido más operativo (costes, ventajas, cualidades). Existe una tercera línea de investigación con un desarrollo más limitado, a la que se adhiere este trabajo y se recoge en el siguiente epígrafe, que aborda el estudio de la eficacia de estas técnicas. El conocimiento previo de la investigación de estos formatos publicitarios ha puesto de manifiesto el interés de los investigadores en com-

\footnotetext{
${ }^{5}$ Las distintas integraciones de los elementos comerciales en los contenidos del medio televisión dan lugar a diferentes tipologías de FNC (BALASUBRAMANIAN, 1994; HACKLEY Y TIWSAKUL, 2006).
} 
prender los procesos que justifican los efectos, en términos de eficacia, que generan las diferentes formas de publicidad en televisión en las audiencias (SHARP ET AL., 2009). El Cuadro 2 resume las principales evidencias empíricas sobre la eficacia de las FNC.

\section{CUADRO 2}

\section{Evidencias empíricas de las FNC}

\begin{tabular}{|c|c|}
\hline Contribución del estudio & Autores \\
\hline $\begin{array}{l}\text { Las FNC, en su conjunto, generan un mayor nivel de recuerdo que la publicidad } \\
\text { convencional (spots). }\end{array}$ & ARRAZOLA ET AL. (2010) \\
\hline $\begin{array}{l}\text { El mayor recuerdo de las FNC se origina en el efecto "sorpresa" que producen en las } \\
\text { audiencias al no estar insertadas en los bloques publicitarios convencionales. }\end{array}$ & $\begin{array}{l}\text { BALASUBRAMANIAN (1994) } \\
\text { RUSELL (2002) } \\
\text { LEE Y FABER (2007) }\end{array}$ \\
\hline Las variables sociodemográficas como el sexo y la edad condicionan el recuerdo de las FNC. & SCHMOLL ET AL. (2006) \\
\hline La complejidad del guión del formato publicitario afecta al recuerdo. & LOWREY (2006) \\
\hline $\begin{array}{l}\text { Se contrasta la relación positiva entre la implicación del consumidor ante la marca y el recuerdo } \\
\text { de la acción de patrocinio. }\end{array}$ & LARDINOIT $(1998,1999)$ \\
\hline $\begin{array}{l}\text { El recuerdo está moderado por la implicación de la audiencia con el producto comunicado y } \\
\text { por el contexto que rodea al formato. }\end{array}$ & De Pelsmacker ET AL. (2002) \\
\hline $\begin{array}{l}\text { El recuerdo de la publicidad está moderado por la congruencia del formato con el programa. } \\
\text { Se producirá un mayor recuerdo cuando la forma publicitaria y el espacio que la acompaña o } \\
\text { en el que se integra, sean congruentes con respecto a los elementos que la audiencia percibe } \\
\text { como felices. }\end{array}$ & LORD Y BURNKRANT (1993) \\
\hline $\begin{array}{l}\text { Las FCN generan un mayor recuerdo, actitudes más positivas y mayores ventas frente a la } \\
\text { publicidad convencional. Se contrasta de forma comparativa entre una telepromoción interna y } \\
\text { un spot. }\end{array}$ & REINARES ET AL. (2007) \\
\hline $\begin{array}{l}\text { El patrocinio televisivo proporciona un mayor recuerdo de marca y aporta mayor prestigio al } \\
\text { anunciante en relación a los spots. }\end{array}$ & REINARES y REINARES (2009). \\
\hline La variable sexo condiciona las actitudes generadas por las FNC. & GUPTA Y GOULD (1997) \\
\hline $\begin{array}{l}\text { La tipología de patrocinio televisivo afecta de forma diferenciada a las actitudes que se generan } \\
\text { en las audiencias. }\end{array}$ & D’Astous y SEGUIN (1999) \\
\hline La integración de una marca en el contenido de un programa influye sobre las actitudes. & $\begin{array}{l}\text { VAN REIJMERSDAL ET AL. } \\
(2007)\end{array}$ \\
\hline $\begin{array}{l}\text { Las asociaciones de los teleespectadores entre los productos emplazados y los personajes que los } \\
\text { utilizan influyen en las actitudes hacia las marcas. }\end{array}$ & $\begin{array}{l}\text { RUSSELL Y STERN (2006) } \\
\text { MATTHES ET AL. (2007) }\end{array}$ \\
\hline $\begin{array}{l}\text { La congruencia entre el emplazamiento de producto y las expectativas del espectador mejoran la } \\
\text { actitud hacia la marca. }\end{array}$ & RuSELL (2002) \\
\hline $\begin{array}{l}\text { La colocación prominente de productos emplazados puede producir actitudes negativas haca la } \\
\text { marca en espectadores con elevados niveles de agrado con los programas utilizados. }\end{array}$ & COWLEY Y BARRON (2008) \\
\hline $\begin{array}{l}\text { Existe una relación inversa en la actitud provocada hacia el producto dependiendo del conocimiento } \\
\text { previo del mismo. El bajo conocimiento previo junto con la elevada participación de la marca en el } \\
\text { programa de televisión tienen un efecto positivo en la actitud hacia el producto. }\end{array}$ & MATTHES ET AL. (2007) \\
\hline
\end{tabular}




\section{CUADRO 2 (Continuación)}

\begin{tabular}{|c|c|}
\hline Contribución del estudio & Autores \\
\hline $\begin{array}{l}\text { Se contrasta la mayor eficacia en términos conductuales de la publicidad no convencional a partir } \\
\text { de la comparación entre una telepromoción interna y un spot. }\end{array}$ & REINARES Y REINARES (2011) \\
\hline La posición preferencial de las FNC produce mejoras conativas. & PARDO (2008) \\
\hline $\begin{array}{l}\text { El emplazamiento de producto se beneficia de la relación positiva entre los espectadores y la } \\
\text { película utilizada en el emplazamiento. }\end{array}$ & REDONDO (2006) \\
\hline $\begin{array}{l}\text { La actitud hacia el product placement varía en función de variables como el género, la edad, la } \\
\text { personalidad y la frecuencia de consumo de películas. }\end{array}$ & GuIDO, ET AL. (2010) \\
\hline $\begin{array}{l}\text { Existe una actitud más favorable a la técnica de emplazamiento de producto en función de la mayor } \\
\text { exposición al medio televisión y a las series utilizadas en el emplazamiento. }\end{array}$ & TORRANO Y FLORES (2005) \\
\hline $\begin{array}{l}\text { La efectividad de los anuncios de televenta está relacionada con el contenido y ejecución del } \\
\text { mensaje. }\end{array}$ & SANZ BLAS (2007) \\
\hline $\begin{array}{l}\text { La efectividad de los anuncios de televenta esta relacionada con la actitud hacia la televenta y la } \\
\text { utilización de canales comerciales sin tienda. }\end{array}$ & $\begin{array}{l}\text { Ruíz MAFÉ Y SAN BLAS } \\
(2007)\end{array}$ \\
\hline $\begin{array}{l}\text { Existe una relación directa entre la identificación del formato publicitario como publicidad por las } \\
\text { audiencias y su menor eficacia. }\end{array}$ & DAHLEN Y EDENIUS (2007) \\
\hline $\begin{array}{l}\text { El emplazamiento de producto es más eficaz sobre el consumo cuando los telespectadores no son } \\
\text { conscientes de su influencia. }\end{array}$ & LAW Y BRAUN (2000) \\
\hline $\begin{array}{l}\text { La eficacia de las FNC está relacionada con la posición que ocupan las marcas publicitarias en } \\
\text { función de la duración temporal del programa que las contiene. }\end{array}$ & GUPTA Y GOULD (2007) \\
\hline $\begin{array}{l}\text { Las FNC (pantalla compartida) reducen el comportamiento del zapping en comparación con los } \\
\text { spots. }\end{array}$ & CHOWDHuRY ET AL. (2007) \\
\hline $\begin{array}{l}\text { Se demuestra que los contenidos de las FNC influyen en la eficacia. Esto se contrasta comparando } \\
\text { los contenidos de un Informercial con los de un spot. }\end{array}$ & HETSRONI y ASYA (2002) \\
\hline $\begin{array}{l}\text { Atribuyen la mayor eficacia de las FNC a su carácter mixto al combinar adecuadamente mensajes } \\
\text { persuasivos y de entretenimiento. }\end{array}$ & HACKLEY Y TIWSAKUL (2006) \\
\hline
\end{tabular}

La mayoría de los trabajos sobre FNC se limitan a aquellos formatos con mayor similitud formal con los anuncios publicitarios convencionales. Desafortunadamente, sólo existe un marco teórico consolidado y conceptualmente relevante sobre Patrocinio televisivo (Hall, 2004; Masterson, 2005, Tiwsakul ET $A L ., 2005)$. A nivel internacional, otros de los formatos investigados son los Infomercials (ElLIOTT Y LOCKARD, 1996; CHAPMAN Y BeLTRAMINI, 2000). Varios investigadores han centrado su atención en el Product placement y la comprensión de cómo utilizar esta forma publi- citaria de forma eficaz en el contexto de televisión (D'Astous y SÉGUIN, 1999; BRENNAN, ET $A L ., 1999$; AVErY y Ferraro, 2000; Russell, 2002; Pervan y Martin, 2002; Russell Y Stern, 2006; MATthes, ET. AL., 2007).

El reducido marco teórico de partida refleja que estamos ante un objeto de estudio del que su potencial no ha transcendido al ámbito académico, a pesar de tratarse de prácticas consolidadas en las estrategias de comunicación de los anunciantes que se están empleando de forma limitada por la falta de comprensión de su funcionamiento (SMIT ET AL., 2009). 


\section{LA EFECTIVIDAD DE LAS FORMAS NO CONVENCIONALES}

La respuesta en términos cognitivos y afectivos de las audiencias a los estímulos publicitarios ha sido un tópico generalizado en la literatura centrada en el campo de la publicidad (Royo Vela, 2005). Este interés se ha trasladado, parcialmente, a la investigación centrada en la publicidad no convencional, que replica los procedimientos para comprender cómo la misma actúa en la memoria y en las actitudes de las audiencias. Sin embargo, son menos frecuentes los trabajos centrados en la componente comportamental de eficacia.

\subsection{Efectividad a nivel cognitivo}

A nivel cognitivo existen antecedentes que reconocen que, bien de forma individual o en su conjunto, las formas de publicidad no convencionales generan, por una parte, elevados niveles de recuerdo, y por otra, un mayor nivel de recuerdo con respecto a la publicidad convencional.

El Patrocinio televisivo, quizás por su mayor afinidad con el Spot, es uno de los formatos en el que se ha analizado sus efectos sobre el recuerdo de las audiencias. Salvo algunas excepciones, como el trabajo de TIWSAKUL ET $A L$. (2005), en el que no se encuentran relaciones positivas en el recuerdo generado por la marca tras su integración en un Patrocinio televisivo, el resto de trabajos encuentran una relación positiva entre su uso, condicionado por determinados factores de influencia, y los efectos cognitivos en las audiencias (WALLISER, 1994; MEIR ET AL., 1997, LARDINOIT, 1998, 1999; MCDANiEl, 1999; MoORe ET AL., 1999).

Por otra parte, algunos trabajos han analizado los factores que favorecen o dificultan el recuerdo en diferentes formatos no convencionales. A este respecto, BALASUBRAMANIAN (1994) concluye afirmando que el mayor recuerdo de las FNC se origina por el efecto sorpresa que las mismas generan en las audiencias al no esperar encontrarse con mensajes comerciales dentro de los programas, o tal y como señala Russell (2002), por no estar insertadas en los bloques publicitarios convencionales. Otros factores moderadores del recuerdo estudiados en la literatura académica han sido el tipo de programa con el que se integra el formato (BLOXHAM, 1998; FURNHAM, ET. AL., 2002; BARTEL-SHEEHAN Y GUO, 2005), la congruencia entre el formato y el programa (LORD Y BURNKRANT, 1993), o la utilización de personajes famosos en el formato (AMOS ET AL., 2008).

Respecto a la cuantificación de dicho recuerdo, de forma comparativa entre Spots y cada uno de los nuevos formatos publicitarios, en el trabajo de ARRAZOLA ET AL. (2010) las diferencias en la probabilidad media de recuerdo oscilan entre 12 puntos y 16 puntos porcentuales. En esta línea de resultados cabe señalar fuentes procedentes de la praxis empresarial. En general, desde el ámbito de la empresa se atribuye mayores niveles que en la literatura académica a las FNC para actuar sobre el recuerdo de las audiencias con respecto a los niveles medios que generan los Spots (IP NeTWORK, 2007; REINARES Y REINARES, 2009). Destacan el trabajo de SCHMID (2003) que cuantifica la diferencia del recuerdo espontáneo de una FNC frente a un Spot entre un 133 y un $188 \%$. Con respecto al sugerido la diferencia a favor de las FNC oscila entre el 70 y el 145\%. En el ámbito español, la investigación de MEdIA PLANNING GROUP (2007) contrasta que los formatos no convencionales se recuerdan un $56 \%$ más que la publicidad convencional (Spots).

\subsection{Efectividad a nivel afectivo}

A nivel afectivo, son escasos los trabajos que han analizado, al margen de los factores de influencia, la eficacia de las FNC. DAHLÉN Y EDENIUS (2007) demuestran que la mayor eficacia de las FNC frente a la publicidad convencional se debe al rechazo de las audiencias de 
los mensajes publicitarios que se identifican explícitamente. Por tanto, los mensajes comerciales que tienen una cierta integración con los contenidos, como sucede con las FNC, generan un menor rechazo (y por lo tanto una mayor eficacia) debido a que las audiencias tienen dificultad para separarlos de los contenidos no publicitarios. A esta misma conclusión llegan MATTHES ET AL. (2007), al demostrar que las actitudes de los individuos hacia las marcas anunciadas a través de las FNC son positivas a pesar de que los individuos no recuerdan haber sido expuestos a un mensaje publicitario. Por el contrario, D'Astous y SÉGUIN (1999), al contrastar los factores ejecucionales de un patrocinio televisivo que condicionan la eficacia del mismo a nivel afectivo, llegan a la conclusión de que se producen actitudes negativas cuando la intencionalidad comercial del formato se oculta mediante sus características formales a la audiencia.

\subsection{Efectividad a nivel conativo}

Respecto a los efectos conativos de las FNC, son todavía menos frecuentes los trabajos existentes en la literatura. Al margen de trabajos que contrastan los efectos en la conducta del Product placement en niños (AUTY Y LeWIS, 2004; HANG Y AUTY, 2008) y jóvenes universitarios, no se tiene constancia de fuentes académicas fuera del ámbito español. Quizás los únicos trabajos sean los de PARDO (2008), que relaciona la mejoras conativas de las FNC con su posición preferencial y, REINARES Y REINARES (2011), en el se presenta un case study que cuantifica la mayor rentabilidad, en términos de llamadas hacia el call center y altas en el servicio, de los formatos no convencionales en relación a una creatividad equivalente en formato Spot. También se pone de manifiesto la mayor eficacia en términos conductuales de la publicidad no convencional en el trabajo desarrollado por REINARES ET AL. (2007) centrado en una Telepromoción interna.

\subsection{Efectividad conjunta}

Finalmente, de forma conjunta para los tres niveles de eficacia propuestos y comparativa con los Spots, el trabajo de REINARES y REINARES (2008) contrasta como las audiencias reconocen una mayor capacidad al patrocinio televisivo para actuar, además de sobre el recuerdo de la marca, sobre las actitudes de las audiencias. Según este trabajo, mientras que el Spot convencional se percibe como un tipo de publicidad que aporta un mayor conocimiento del producto, las caretas de Patrocinio se perciben como formatos que permiten recordar mejor la marca anunciada y aportan mayor prestigio al anunciante. Por otro lado, los dos tipos de publicidad se conciben como formatos que ayudan a la venta del producto anunciado por igual.

\subsection{Formulación de hipótesis}

Tras esta revisión, se puede concluir que en la literatura académica la mayor eficacia de las FNC frente a los Spots, a excepción del trabajo de Arrazola eT AL. (2010), referido al conjunto de FNC, sólo se ha contrastado, de forma exploratoria y experimental, en una minoría concreta de formatos no convencionales. Son trabajos que comparan la eficacia que generan los Spots frente a los Infomercials (SINGH ET $A L ., 2000$; HetSRONI y ASYA, 2002; LAFERLE Y EDWARDS, 2006), frente a los Patrocinios (REINARES Y REINARES, 2009) o frente a las Telepromociones internas dentro de un programa (RUSSELL, 2002; REINARES ET AL., 2007).

A pesar de la dificultad de generalizar al conjunto de FNC, resultados asociados a tipologías especificas, la comparación entre la eficacia de diferentes FNC y el Spot pone de manifiesto la mayor eficacia de las FNC. Estos antecedentes hacen coherente formular hipótesis que recojan el carácter positivo de la eficacia en términos cognitivos, afectivos y de conducta generada por la Telepromoción externa frente a los Spots de la siguiente manera: 
H1. La Telepromoción externa es más eficaz que la publicidad convencional (Spots).

\section{Hla: La Telepromoción externa es más eficaz en términos cognitivos que la publicidad convencional (Spots).}

H1b: La Telepromoción externa es más eficaz en términos afectivos que la publicidad convencional (Spots).

H1c: La Telepromoción externa es más eficaz en términos conativos que la publicidad convencional (Spots).

\section{FACTORES QUE INFLUYEN EN LA EFICACIA PUBLICITARIA}

La fragmentación de las audiencias y la especialización de las cadenas y sus contenidos, entre otros factores, han generado una mayor preocupación por mejorar la eficacia de la publicidad en función del perfil del público objetivo al que se dirige.

En la literatura sobre eficacia se pueden encontrar múltiples trabajos tendentes a analizar la influencia de variables relativas al individuo en la eficacia de la publicidad convencional, siendo muy escasos, por no decir inexistentes, los trabajos de esta índole realizados en el contexto de las FNC. Es por ello que en este trabajo también se ha querido abordar el análisis de cómo influye una serie de variables personales en la eficacia de la publicidad no convencional. Más concretamente, se analizará la influencia de las variables sociodemográficas relativas al género, edad y clase social. Ahora bien, para poder plantear las hipótesis de trabajo hemos de señalar que para aquellas variables y niveles de eficacia en los que no existen antecedentes teóricos en formatos no convencionales, se ha tomado como antecedente en dicha formulación de hipótesis el contexto de la publicidad convencional.

\subsection{Influencia del género de la audiencia en la eficacia publicitaria}

En el medio televisión son limitados los antecedentes teóricos que relacionan la variable género con los efectos publicitarios a nivel cognitivo y asumen la existencia de escasas diferencias en el recuerdo en función de dicha característica. Tampoco queda demostrada esta relación en el trabajo de ARRAzOLA ET $A L$. (2010) para el conjunto de formatos no convencionales.

Los trabajos centrados en esta variable han analizado principalmente las diferencias que origina el género en la publicidad en lo que a las actitudes se refiere. De esta forma, estudios como los de HeEter y GreEnBerg (1985) o SMith (1993) han demostrado diferencias entre hombres y mujeres a la hora de valorar los contenidos del medio televisión y procesar sus contenidos publicitarios (DARLEY Y SMITH, 1995). Tal y como sugieren estos autores, es razonable asumir que estas diferencias son trasladables a los mensajes comerciales en televisión. En el ámbito de la publicidad no convencional sólo se ha contrastado la influencia del género en la eficacia publicitaria en el formato de emplazamiento de producto (GUPTA Y Gould, 1997).

Así se puede argumentar que, al no disponer de un soporte científico que nos haga suponer que en un formato se producen resultados distintos al otro, la especialización de la oferta televisiva por géneros, requiere abordar de forma profunda este análisis. Por ello resulta viable proponer una hipótesis general que reconozca la eventual influencia de la variable género en los distintos niveles de efectos de la publicidad en el entorno de televisión. Esta hipótesis quedaría expresada de la siguiente forma:

H2: El género influye en la eficacia de la publicidad 


\subsection{Influencia de la edad de la audiencia en la eficacia publicitaria}

Gran parte de los trabajos centrados en los efectos de la edad sobre la eficacia de los mensajes publicitarios, han tratado de comprender los procesos cognoscitivos en niños o jóvenes. Sin embargo, el progresivo envejecimiento de la población, y a su vez de las audiencias del medio de televisión, justifica la necesidad de completar el estudio de esta relación en rangos de edad más amplios (DroULERS, 2006).

Dubow (1994) y Bijmolt ET AL. (1998) justifican la influencia de la edad en los procesos cognoscitivos. Así, el primero demuestra que el reconocimiento de marca que generan los Spots decrece con la edad. Para el conjunto de FNC, REINARES (2010) concluye también que al aumentar la edad disminuye el recuerdo, aunque cada vez en menor proporción.

Respecto a la componente actitudinal de la eficacia, De Pelsmacker ET AL. (2005a) encuentran un efecto moderador de la edad sobre las actitudes hacia formatos no convencionales de publicidad. Cabe destacar el trabajo de Phillips y Stanton (2004) por las interesantes correlaciones que demuestran entre la componente afectiva y de conducta y el recuerdo en función de la edad. Si bien los resultados son coincidentes con otras fuentes al establecer un mayor recuerdo en los rangos de edad 18-35 años y los perfiles de audiencia a partir de 65 años, hay un sentido contrario entre el recuerdo y la componente comportamental de eficacia. Así el mayor recuerdo entre el target joven genera una menor intención de compra de la marca recordada. Esta relación se invierte en el target mayor de 65 años.

En general todos los trabajos consultados encuentran diferencias significativas en el recuerdo en función de la edad. Sin embargo, al analizar los resultados en otros niveles de eficacia, se encuentran ciertas discrepancias que refuerzan el interés académico de una hipótesis como la que se plantea a continuación:
H3: La edad influye en la eficacia publicitaria.

\subsection{Influencia de la clase social de la audiencia en la eficacia publicitaria}

Respecto a las variables que conforman la clase social de los individuos, en el ámbito de la publicidad convencional se ha demostrado que el nivel cultural afecta a la eficacia en términos cognoscitivos de los mensajes publicitarios (VALKEMBURG Y BUIJZEN, 2005). En trabajos desarrollados en el entorno de la publicidad no convencional, en la investigación sobre los efectos cognoscitivos de las FNC de REINARES (2010) se aprecian, en el conjunto de FNC, que existe un mayor recuerdo en las clases sociales baja y media-baja frente al resto de perfiles sociales. En este sentido, autores como DAHLÉN Y EDENIUS (2007) demuestran que la identificación del carácter persuasivo de los mensajes publicitarios condiciona mecanismos de protección en las audiencias ante dichos mensajes. En los niveles culturales altos se identifica más fácilmente los mensajes persuasivos entre los programas, sean o no explícitos. Sobre la base de estos resultados se plantea la siguiente hipótesis de trabajo:

\section{H4: La clase social influye en la eficacia de la publicidad}

\section{METODOLOGÍA DE INVESTIGACIÓN}

Como se ha señalado en la introducción, dada la complejidad técnica de la investigación publicitaria en un entorno real, son escasos los estudios que han analizado de forma comparativa las diferencias de eficacia entre los anuncios de televisión convencionales y los nuevos formatos publicitarios. De acuerdo con autores como Moorman ET AL. (2007) y Pieters y BiJMOLT (1997), el proceso metodológico seguido en la presente investigación trata de superar, en 
la medida de lo posible, algunas de las limitaciones de los trabajos consultados.

En este contexto, una de las aportaciones metodológicas de este estudio es analizar en un entorno real la existencia o no de diferencias de eficacia entre un formato convencional (Spot) y uno no convencional (Telepromoción externa).

Respecto al ámbito geográfico de estudio, se ha elegido España como el mercado idóneo para el mismo. España es el quinto mercado publicitario de televisión en Europa, y ocupa el segundo lugar, después de Italia, en términos de la participación de los medios de comunicación en los gastos totales de publicidad en poder de la televisión (ARRAzolA, et. al., 2010). Sus anunciantes invierten mayoritariamente en publicidad televisiva, y tienen una mayor historia y desarrollo en la adopción de los nuevos formatos frente a otros mercados europeos (CARAT, 2005). Es previsible que muchos de estos formatos de publicidad, de uso común en España, se desarrollen a corto plazo en los países en los que las estrategias publicitarias son más conservadoras en la actualidad (CARAT CRISTAL y BIRD \& Bird, 2002; OfCOM, 2005).

La aplicación de la investigación en un entorno real hizo necesaria la colaboración de un soporte televisivo (Telecinco, cadena generalista que emite en abierto y con una cuota media de pantalla que se sitúa alrededor del $15 \%$ ) para la difusión en una franja horaria de prime time (con una cuota de pantalla según datos de Sofrés del 28,6\%) del Spot y de la Telepromoción seleccionados.

Los mensajes comerciales objeto de estudio fueron: el Spot de 20 segundos de duración de Pizzas "Micro" de la marca "Buittoni" y la Telepromoción de 65 segundos de contrato de telefonía móvil del Plan "Total" de precios de la marca "Vodafone", asociada creativamente a la teleserie "Los Serrano"6 (véase Figura 1). En el diseño de la investigación se trató de neutralizar determinadas variables consideradas moderadoras del recuerdo generado (LAMAS, 2004). El criterio principal en el diseño fue que ambos formatos, Spot y Telepromoción, se refirieran a un producto o servicio nuevo y que su creatividad no hubiese tenido difusión previa en televisión y fueran transmitidos por un canal único, de manera que no hubiese ninguna influencia en la componente cognoscitiva de eficacia de las campañas de conocimientos previos del producto o la campañas. Por otra parte los objetivos de ambos formatos se dirigían a dar a conocer la oferta y sus características.

\footnotetext{
${ }^{6}$ La Telepromoción se asociaba a la teleserie "Los Serrano", que es una Sitcom familiar de audiencia generalista, y se emitió cómo único mensaje comercial, situado entre un resumen de capítulos anteriores de la teleserie, de 6 minutos de duración, y el inicio del capítulo nuevo. Mientras que el Spot se situó en segunda posición en el bloque publicitario, de 4 minutos de duración, anterior a la emisión del resumen de la teleserie.
} 


\section{Anuncio PIZZA BUITONI MICRO}
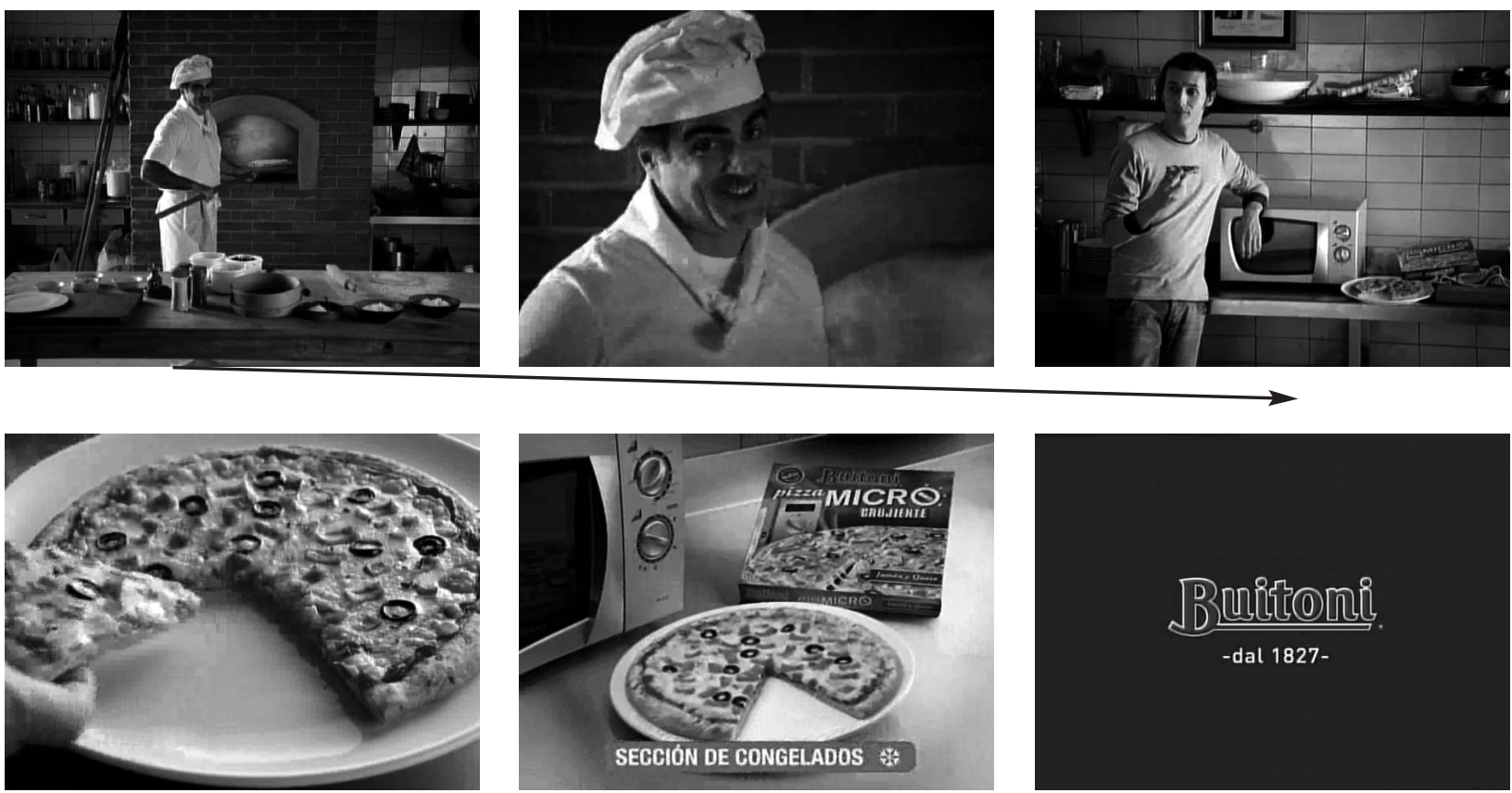

Telepromoción VODAFONE
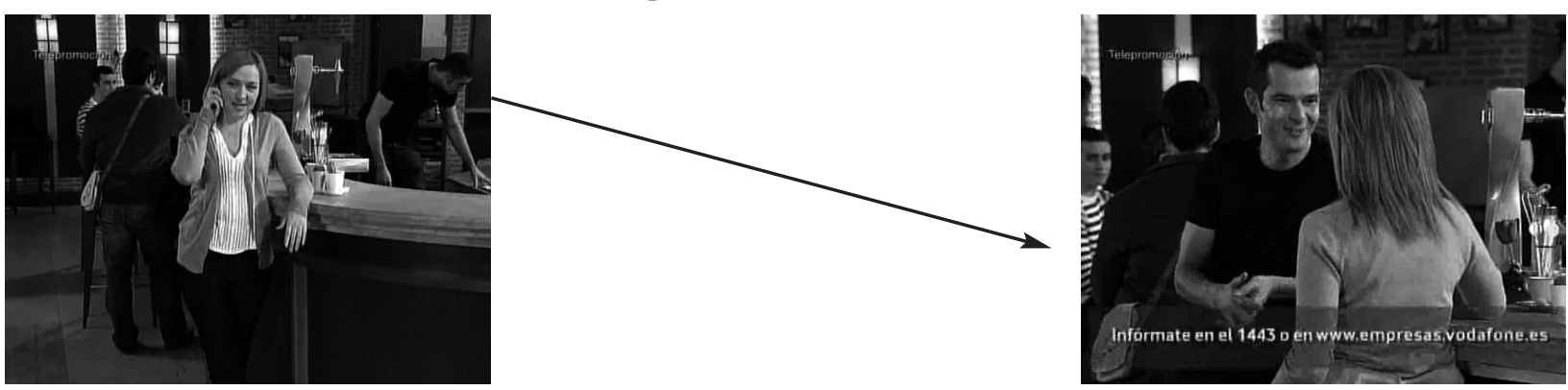

En la acción vemos a EMILIA, propietaria de la taberna de LOS SERRANO sentada en la barra, frente a un café. Un proveedor llega con un barril de cerveza en una mano y un móvil en la otra. Emilia y el proveedor hablan de que otra de las ventajas del Plan Total para Autónomos de Vodafone es que tienes el número fijo y el móvil sin desvios. Plan Vodafone. Por $25 €$ al mes llamadas a fijos nacionales, llamadas entre los móviles de mi negocio y llamadas al número favorito Vodafone que elija. Y también tengo el número fijo del bar en el móvil sin desvíos. Además, un Nokia E50 con correo móvil por 19 euros.

El universo lo constituyeron individuos a partir de 10 años y residentes en Madrid. Este universo poblacional se corresponde con la audiencia de los programas donde se encontraban insertados los formatos publicitarios. La selección muestral se realizó en los hoga- res de los propios encuestadores utilizando un muestreo por relaciones. De forma complementaria se aplicaron cuotas con afijación proporcional al género y edad. El Cuadro 3 resume el proceso metodológico seguido en la investigación. 
CUADRO 3

\section{Ficha técnica del proceso metodológico}

\begin{tabular}{|l|l|}
\hline Procedimiento metodológico & Encuestas personales a través de cuestionario estructurado \\
\hline Universo & Individuos mayores de 10 años. \\
\hline Ámbito & Madrid \\
\hline Muestra real & 816 \\
\hline Error muestral & $+/-3,4$ \\
\hline Nivel de confianza & $95 \%(\mathrm{Z}=1,96)$ \\
\hline Varianza máxima admitida & $\mathrm{P}=\mathrm{q}=50 \%$ \\
\hline
\end{tabular}

Los encuestados que formaron parte de la muestra fueron aquellos que estuvieron expuestos a la cadena durante el tiempo completo de emisión de la parrilla en la que se emitieron tanto el Spot como la Telepromoción. Los encuestadores estaban debidamente formados en el procedimiento a seguir en la recogida de información.

Los indicadores de medidas de eficacia utilizados han sido los usados habitualmente en la investigación científica. Así, para cuantificar el componente cognitivo se han utilizado los recuerdos espontáneo, sugerido y verificado de los contenidos del anuncio (BEERLI Y MARTíN, 1998; NorRis ET AL, 2003; FurnhaM ET AL., 2002), además de un test de reconocimiento visual SÍ/NO de los anuncios. Una de las razones que justifican el uso tanto del recuerdo como del reconocimiento es el hecho de que en los niños se requiere la utilización del reconocimiento como medida de eficacia al ser una medida más sensible y, por tanto, con un mayor poder para captar si el anuncio ha dejado o no alguna huella en la mente del espectador. Por otra parte, al utilizar productos y campañas nuevas, las medidas de reconocimiento permiten constatar con mayores garantías si los estímulos publicitarios han dejado alguna huella cognitiva en la mente de los individuos al tratarse de una medida más sensible que el recuerdo. Respecto al nivel afectivo se ha utilizado como medida de eficacia la actitud hacia el anuncio, la cual se ha desarrollado a partir de los ítems de escalas de medida validadas en estudios anteriores (AAKER Y BRUZZONE, 1981; MoldOVAN, 1985; ZINKHAN y FoRNELL, 1985; MCKeNZIE ET AL., 1986; EdELl y BURKE, 1987; HoMer, 1990; MAdDEN ET AL., 1998; REINARES ET AL., 2007). Por último, para evaluar el nivel conativo se utilizó la intención de compra de los productos anunciados en los dos formatos analizados, siguiendo la pauta de las escalas de intencionalidad de compra recogidas en el trabajo de BeERLi Y MARTín (1999). En el Cuadro 4 se recogen las medidas y escalas multi-ítem utilizadas en este trabajo, que se corresponden concretamente con las siguientes:

- Recuerdo espontáneo de la categoría de producto, marca y características de los anuncios. Se utilizaron como indicadores de la calidad del recuerdo de las características de los formatos publicitarios: Recuerdo relacionado específico, relacionado general, total relacionado, no relacionado y sin contenido. A partir de la información obtenida, se creó una nueva variable que se ha etiquetado como "Intensidad del recuerdo espontáneo", cuyos estados de la variable se recogen en dicho Cuadro.

- Recuerdo sugerido de la marca y características del anuncio sugiriendo categoría de producto. Se utilizaron de nuevo como 
indicadores de la calidad del recuerdo de las características de los formatos publicitarios: Recuerdo relacionado específico, relacionado general, total relacionado, no relacionado y sin contenido. Al igual que para el recuerdo espontáneo, en este caso también se creó una nueva variable que se ha etiquetado como "Intensidad del recuerdo sugerido", cuyos estados de la variable se recogen en el Cuadro 4.

- Reconocimiento visual SI/NO de los formatos, utilizándose además una escala de un único ítem tipo Likert de 7 puntos que mide la seguridad del reconocimiento.
- Liking, a través de una escala de un único ítem tipo Likert de 7 puntos para indicar la intensidad de agrado hacia el anuncio.

- Actitud hacia el mensaje, utilizando una escala tipo Likert de 7 puntos y 6 ítems (véase Cuadro 4).

- Actitud hacia el formato publicitario, utilizando una escala tipo Likert de 7 puntos y 9 ítems (véase Cuadro 4).

- Intención de compra, utilizando una escala de un único ítem tipo Likert de 7 puntos que mide el grado en que se comprarían en la próxima compra las marcas de los productos anunciados.

\section{CUADRO 4 \\ Ítems definitivos de las escalas de medida utilizadas}

\begin{tabular}{|c|c|}
\hline \multicolumn{2}{|c|}{ Intensidad del Recuerdo Espontáneo } \\
\hline 1 & No se recuerda nada \\
\hline 2 & Sólo se recuerda la categoría de producto \\
\hline 3 & Se recuerda categoría o marca del producto anunciado y se describe genéricamente el anuncio \\
\hline 4 & Se recuerda categoría y marca del producto anunciado y se describe genéricamente el anuncio \\
\hline 5 & Se recuerda categoría o marca del producto anunciado y se describe específicamente el anuncio \\
\hline 6 & Se recuerda categoría y marca del producto anunciado y se describe específicamente el anuncio \\
\hline \multicolumn{2}{|c|}{ Intensidad del Recuerdo Sugerido } \\
\hline 1 & No se recuerda ningún anuncio de la categoría de producto sugerida \\
\hline 2 & $\begin{array}{l}\text { Se recuerda la existencia de un anuncio de la categoría de producto sugerida, pero no se recuerda ni la } \\
\text { marca anunciada ni se describe nada del anuncio }\end{array}$ \\
\hline 3 & $\begin{array}{l}\text { Se recuerda la existencia de un anuncio de la categoría de producto sugerida, pero no se recuerda la } \\
\text { marca anunciada, aunque se describe genéricamente el anuncio }\end{array}$ \\
\hline 4 & $\begin{array}{l}\text { Se recuerda la existencia de un anuncio de la categoría de producto sugerida, se recuerda la marca } \\
\text { anunciada y se describe genéricamente el anuncio o no se dice nada del mismo }\end{array}$ \\
\hline 5 & $\begin{array}{l}\text { Se recuerda la existencia de un anuncio de la categoría de producto sugerida, pero no se recuerda la } \\
\text { marca anunciada, aunque se describe específicamente el anuncio }\end{array}$ \\
\hline 6 & $\begin{array}{l}\text { Se recuerda la existencia de un anuncio de la categoría de producto sugerida, se recuerda la marca } \\
\text { anunciada y se describe específicamente el anuncio }\end{array}$ \\
\hline \multicolumn{2}{|c|}{ Actitud hacia el mensaje publicitario } \\
\hline & Es un mensaje atractivo \\
\hline & Es un mensaje creíble \\
\hline & Es un mensaje original \\
\hline & Es un mensaje informativo \\
\hline
\end{tabular}




\section{CUADRO 4 (Continuación)}

Es un mensaje fácil de recordar

Es un mensaje fácil de comprender

Actitud hacia el formato

Este mensaje capta mi atención

El objetivo de este mensaje es entretener

El objetivo de este mensaje es proporcionar información

El objetivo de este mensaje es vender el producto

Este tipo de mensajes resultan engañosos

Este tipo de mensajes resultan muy convincentes

No me gusta que interrumpan el programa con este tipo de mensajes

Este mensaje es un anuncio publicitario

Este mensaje se confunde con el programa en el que aparece

Las características de la muestra utilizada se recogen en la Tabla 1.

TABLA 1

Distribución de la muestra

\begin{tabular}{|l|c|c|l|c|c|}
\hline \multicolumn{1}{|c|}{ CARACTERÍSTICAS } & N & \% & CARACTERÍSTICAS & N & \% \\
\hline GÉNERO & & & EDAD & & \\
\hline Hombre & 319 & 39.0 & De 10 a 14 años & 38 & 4.6 \\
\hline Mujer & 497 & 60.8 & De 15 a 19 años & 69 & 8.4 \\
\hline NIVEL DE ESTUDIOS & & & De 20 a 24 años & 173 & 21.1 \\
\hline Sin estudios & 33 & 4.0 & De 25 a 34 años & 149 & 18.2 \\
\hline Primarios & 96 & 11.7 & De 35 a 44 años & 42 & 5.1 \\
\hline Secundarios & 151 & 18.5 & De 45 a 54 años & 203 & 24.8 \\
\hline Bachiller & 231 & 28.2 & De 55 a 64 años & 108 & 13.2 \\
\hline Universitarios medios & 151 & 18.5 & 65 años o más & 34 & 4.2 \\
\hline Universitarios superiores & 155 & 18.9 & $\begin{array}{l}\text { CONSUMO DE } \\
\text { TELEVISIÓN }\end{array}$ & & \\
\hline CLASE SOCIAL & & & Una hora o menos & 125 & 15.3 \\
\hline Alta & 112 & 13.7 & Entre 1 y 2 horas & 256 & 31.3 \\
\hline Media-alta & 180 & 22.0 & Entre 2 y 3 horas & 222 & 27.1 \\
\hline Media-media & 358 & 43.8 & Entre 3 y 4 horas & 112 & 13.7 \\
\hline Media-baja y baja & 74 & 9.0 & Entre 4 y 5 horas & 50 & 6.1 \\
\hline Baja & 2 & 0.2 & Más de 5 horas & 47 & 5.7 \\
\hline
\end{tabular}




\section{ANÁLISIS DE RESULTADOS Y DISCUSIÓN}

\subsection{Influencia del tipo de formato en las medidas de eficacia publicitaria}

Antes de proceder a realizar los análisis pertinentes para el cumplimiento de los objetivos que se plantean con la presente investigación, se procedió a analizar la validez y fiabilidad de la escala de actitud hacia el anuncio utilizada en el estudio, que aglutinaba los ítems de las escalas de actitud hacia el mensaje y hacia el formato. A tal objeto, se aplicó, en primer lugar, un análisis factorial exploratorio para depurar y conocer la naturaleza dimensional de dicha escala; en segundo lugar, un análisis factorial confirmatorio con el propósito de confirmar los resultados obtenidos, utilizando para ello ecuaciones lineales estructurales, y, finalmente, el coeficiente de fiabilidad compuesta y el análisis de la varianza extraída para evaluar su fiabilidad, cuyos niveles son aceptables. En la Tabla 2 se recogen los resultados del análisis factorial confirmatorio, pudiéndose comprobar que los indicadores de bondad del ajuste son aceptables, dado que todas las medidas de ajuste absoluto, incremental y de parsimonia se sitúan alrededor de los límites recomendados en la literatura y que todos los pesos de regresión estandarizados presentan razones críticas superiores al valor recomendado de +1,96. Como se puede apreciar, dichos resultados ponen de manifiesto que la actitud hacia el anuncio es de naturaleza multidimensional, integrada por cuatro componentes relacionados con el atractivo, el carácter informativo, la credibilidad y el grado de molestia.

TABLA 2

Resultados del modelo de medida de la actitud hacia el anuncio

\begin{tabular}{|c|c|c|c|c|c|}
\hline \multicolumn{3}{|l|}{ Relaciones causales } & Estimadores & Razones & $\boldsymbol{P}$ \\
\hline Es un mensaje atractivo & $<---$ & Atractivo & 0.775 & & \\
\hline Es un mensaje original & $<---$ & Atractivo & 0.701 & 27.010 & 0.000 \\
\hline Este mensaje capta mi atención & $<---$ & Atractivo & 0.784 & 30.079 & 0.000 \\
\hline El objetivo de este mensaje es entretener & $<---$ & Atractivo & 0.428 & 16.167 & 0.000 \\
\hline Es un mensaje fácil de recordar & $<---$ & Atractivo & 0.602 & 23.045 & 0.000 \\
\hline Es un mensaje creíble & $<---$ & Credibilidad & 0.751 & & \\
\hline Este tipo de mensajes resultan muy convincentes & $<---$ & Credibilidad & 0.694 & 24.349 & 0.000 \\
\hline Es un mensaje informativo & $<---$ & Informativo & 0.859 & & \\
\hline El objetivo de este mensaje es proporcionar información & $<---$ & Informativo & 0.768 & 18.829 & 0.000 \\
\hline Este tipo de mensajes resultan engañosos & $<---$ & Molestia & 0.589 & & \\
\hline $\begin{array}{l}\text { No me gusta que interrumpan el programa con este tipo de } \\
\text { mensajes }\end{array}$ & $<---$ & Molestia & 0.284 & 6.132 & 0.000 \\
\hline Este mensaje se confunde con el programa en el que aparece & $<---$ & Molestia & 0.203 & 4.966 & 0.000 \\
\hline \multicolumn{6}{|l|}{ Indicadores de bondad de ajuste } \\
\hline \multicolumn{6}{|c|}{$\mathrm{CMIN}=451.861(\mathrm{p}=0.000), \mathrm{CMIN} / \mathrm{DF}=9.414, \mathrm{CFI}=0.926, \mathrm{RMSEA}=0.072, \mathrm{TLI}=0.879, \mathrm{NFI}=0.918}$, \\
\hline \multicolumn{6}{|l|}{$\mathrm{PCFI}=0.570, \mathrm{PNFI}=0.565, \mathrm{AIC}=535.534$} \\
\hline \multicolumn{6}{|l|}{ Indicadores de fiabilidad } \\
\hline $\begin{array}{l}\text { Fiabilidad compuesta }=0.889 \\
\text { Varianza extraída }=0.425\end{array}$ & & & & & \\
\hline
\end{tabular}


Para poder contrastar las hipótesis relativas a las medidas cognoscitivas, se ha procedido a la realización de un análisis de la chi-cuadrado al objeto de conocer la existencia o no de diferencias significativas en los niveles de recuerdo alcanzados por el formato convencional frente al no convencional. Los resultados de este análisis, que se presentan en la Tabla 3, ponen de manifiesto claramente que existen importantes diferencias en todas las medidas cognoscitivas de eficacia utilizadas en la presente investigación, siendo el formato no convencional el que registra mayores tasas de recuerdo y reconocimiento. Sobre la base de los resultados se acepta la hipótesis H1a en la que se establecía que la Telepromoción era más eficaz en términos cognoscitivos que los Spots.

Estos resultados son congruentes con los escasos trabajos con objetivos similares centrados en otras formas no convencionales (Schmid, 2003; Media Planning Group, 2007; Reinares ET. AL. 2007; Arrazola ET $A L ., 2010)$. Pueden deberse a las asociaciones que los telespectadores han hecho de la Telepromoción con la serie vinculada a la misma, así como a los efectos positivos derivados de esta integración tal y como han propuesto algunos autores como SPECK Y ELLIOTT (1997); HETSRONI y ASYA (2002); LOWREY (2006); Moorman ET AL. (2007) o SMit ET AL. (2009). Otra posible explicación la podemos encontrar en los trabajos de HACKLEY Y TIW-
SAKUL (2006) y MASTERSON (2005), en los que se demuestra que los formatos no convencionales generan mayores niveles de recuerdo debido precisamente a su mayor capacidad para entretener y captar la atención. Además se podría justificar este mayor recuerdo por el efecto "sorpresa" propuesto, entre otros, por BALASUBRAMANIAN (1994), RusSELl (2002) o LEE Y FABER (2007), derivado de su ubicación fuera del entorno habitual de los mensajes publicitarios (bloque publicitario). Finalmente, y extrapolando los resultados de los trabajos de Fabian (1986), Singh y Cole (1993) o BigNÉ Y MiQuel (1996), en los que se ha demostrado que los Spots de mayor duración son capaces de generar un mayor recuerdo, la mayor duración de la Telepromoción utilizada, 65 segundos frente a los 20 segundos del Spot, puede contribuir al mayor recuerdo de la primera. Así como el hecho de que el anuncio convencional aparece en un bloque publicitario junto con otros anuncios, influyendo negativamente en el recuerdo (WEBB y RAY, 1979; PIETERS Y BIJMOLT, 1997), frente a la nula competencia que tiene en su bloque la Telepromoción. De la misma manera, la posición preferente respecto al programa que ocupa la Telepromoción frente al Spot, puede tener un efecto primacía directamente relacionado con el mayor recuerdo, tal y como contrastan autores como MoOrman ET AL. (2005), GUPTA Y GOULD (2007) y Li (2010). 
TABLA 3

Influencia del tipo de anuncio en las medidas cognoscitivas de eficacia

\begin{tabular}{|c|c|c|c|c|c|c|}
\hline \multirow{2}{*}{ MEDIDAS COGNOSCITIVAS } & \multirow{2}{*}{ CATEGORÍA } & \multicolumn{2}{|c|}{ Spot } & \multicolumn{2}{|c|}{ Telepromoción } & \multirow{2}{*}{$\begin{array}{l}\chi^{2} \\
(\mathbf{p})\end{array}$} \\
\hline & & $\mathbf{N}$ & $\%$ & $\mathbf{N}$ & $\%$ & \\
\hline \multirow{2}{*}{ Recuerdo espontáneo de la categoría de producto } & Sí & 81 & 9.9 & 268 & 32.8 & \multirow{2}{*}{$\begin{array}{l}127.369 \\
(0.000)\end{array}$} \\
\hline & No & 737 & 90.1 & 550 & 67.2 & \\
\hline \multirow{2}{*}{ Recuerdo espontáneo de la marca } & Sí & 64 & 7.8 & 199 & 24.3 & \multirow{2}{*}{$\begin{array}{l}82.570 \\
(0.000)\end{array}$} \\
\hline & No & 754 & 92.2 & 619 & 75.7 & \\
\hline \multirow{2}{*}{$\begin{array}{l}\text { Recuerdo espontáneo de la categoría de producto y de la } \\
\text { marca }\end{array}$} & Sí & 63 & 7.7 & 198 & 24.2 & \multirow{2}{*}{$\begin{array}{l}83.082 \\
(0.000)\end{array}$} \\
\hline & No & 755 & 92.3 & 620 & 75.8 & \\
\hline \multirow{2}{*}{ Recuerdo sugerido de la marca } & Sí & 110 & 14.6 & 220 & 35.5 & \multirow{2}{*}{$\begin{array}{l}81.639 \\
(0.000)\end{array}$} \\
\hline & No & 645 & 85.4 & 400 & 64.5 & \\
\hline \multirow{2}{*}{ Reconocimiento del anuncio } & Sí & 287 & 44.5 & 264 & 66.0 & \multirow{2}{*}{$\begin{array}{l}45.803 \\
(0.000)\end{array}$} \\
\hline & No & 358 & 55.5 & 136 & 34.0 & \\
\hline
\end{tabular}

Como se puede observar en la Tabla 3, del total de la muestra de individuos encuestados y en lo que a las medidas de recuerdo espontáneo se refiere, se puede decir que (1) tan sólo el $9.9 \%$ recuerda haber visto un anuncio de pizzas frente al $32.8 \%$ que manifiesta recordar haber visto un anuncio de un plan de precios de contrato de telefonía móvil; (2) tan sólo el 7.8\% recuerda la marca Buitoni frente al $24.3 \%$ que recuerda haber viso una anuncio de la marca Vodafone, y, finalmente, (3) sólo un $7.7 \%$ es capaz de recordar correctamente la categoría de producto y la marca anunciada en el formato convencional frente al $24.2 \%$ en el formato no convencional. Estos mismos resultados tan dispares a favor del formato no convencional se producen en el recuerdo sugerido de marca (14.6\% y $35.5 \%$ para el formato convencional y el no convencional, respectivamente) y también en el reconocimiento ( $44.5 \%$ y $66.0 \%$ para el formato convencional y el no convencional, respectivamente).

Sin embargo, y a pesar de que el formato no convencional favorece los niveles de recuerdo, en la Tabla 4 se aprecia como la calidad del recuerdo es algo superior en el formato convencional, ya que del total de individuos que ha recordado tanto la categoría de producto como la marca anunciada, el $38.1 \%$ y el $28.6 \%$, ha sido capaz de describirlo en su totalidad o partes específicas del anuncio, respectivamente, frente al $32.3 \%$ y $18.7 \%$ del formato no convencional. De hecho, el porcentaje de individuos que no es capaz de describirlo es muy superior en el formato no convencional $(25.3 \%)$ frente al convencional $(7.9 \%)$. Estos mismos resultados se reproducen en el recuerdo sugerido.

En el estudio comparativo entre Spots y nuevos formatos publicitarios en televisión de SCHMID (2003), se obtienen resultados similares sobre el mayor recuerdo pero de peor calidad generado por los nuevos formatos. Se puede extraer una posible interpretación de este resultado a partir de los trabajos de LOWREY (2006) y RomANiUK ET AL. (2009), en los que se pone de manifiesto que formatos con menor duración, o como señala el estudio de RTL Nederland sobre formatos que tienen una mayor repetición (BACKSTAGE, 2008), generan un mayor aprendizaje en los telespectadores. 
Finalmente, es de resaltar que en lo que al reconocimiento se refiere, se observan unas tasas de seguridad en la respuesta mayores en el formato no convencional frente al convencional. Así, del total de individuos que han manifestado reconocer el anuncio que se les ha mostrado, el $34.5 \%$ ha manifestado total seguridad en su respuesta en el caso del formato convencional frente al $52.1 \%$ para el formato no convencional. Estos resultados nos llevan a pensar que el formato no convencional es capaz de imprimir una huella más profunda en el individuo que el convencional.

TABLA 4

Nivel de descripción de los anuncios para las medidas de recuerdo

\begin{tabular}{|l|c|c|c|c|c|c|c|c|}
\hline \multirow{2}{*}{$\begin{array}{c}\text { NIVELES DE DESCRICIÓN } \\
\text { DEL ANUNCIO }\end{array}$} & \multicolumn{3}{|c|}{ Recuerdo Espontáneo } & \multicolumn{4}{c|}{ Recuerdo Sugerido } \\
\cline { 2 - 10 } & \multicolumn{2}{|c|}{ Spot } & \multicolumn{2}{c|}{ Telepromoción } & \multicolumn{2}{c|}{ Spot } & \multicolumn{2}{c|}{ Telepromoción } \\
\cline { 2 - 10 } & $\mathbf{N}$ & $\mathbf{\%}$ & $\mathbf{N}$ & $\mathbf{\%}$ & $\mathbf{N}$ & $\mathbf{\%}$ & $\mathbf{N}$ & $\mathbf{\%}$ \\
\hline Todo & 24 & 38.1 & 64 & 32.3 & 43 & 39.1 & 56 & 25.5 \\
\hline Parte general & 14 & 22.2 & 39 & 19.7 & 21 & 19.1 & 42 & 19.1 \\
\hline Parte específica & 18 & 28.6 & 37 & 18.7 & 28 & 25.5 & 45 & 20.5 \\
\hline Sin relación & 8 & 3.2 & 8 & 4.0 & 5 & 4.5 & 8 & 3.6 \\
\hline Nada & 5 & 7.9 & 50 & 25.3 & 13 & 11.8 & 69 & 31.4 \\
\hline TOTAL & 63 & 100.0 & 198 & 100.0 & 110 & 100.0 & 220 & 100.0 \\
\hline
\end{tabular}

Por otra parte, y para poder contrastar las hipótesis relativas a las medidas afectivas y conativas, se ha procedido a la realización de un análisis de diferencias de medias, al objeto de conocer la existencia o no de diferencias significativas en el liking, en cada una de las dimensiones de la actitud hacia el anuncio y en la intención de compra entre el Spot y la Telepromoción. Los resultados de este análisis, que se presentan en la Tabla 5, ponen de manifiesto que (1) existen diferencias significativas en el nivel de liking entre ambos formatos, siendo el formato convencional el que goza de un mayor agrado por parte de los individuos encuestados (3.94 frente a 3.63); (2) el formato convencional se percibe como más atractivo frente al no convencional (3.57 frente a 3.43), siendo considerado en general como más atractivo, original, entretenido, fácil de recordar y con una mayor capacidad para captar la atención; (3) el formato no convencional es el que se considera como más informativo (5.57 frente a 4.17); (4) no existen diferencias en los niveles de credibilidad de ambos tipos de formato, resultando igualmente convincentes; (5) el formato no convencional se percibe como más engañoso, interrumpiendo la programación televisiva y confundiéndose incluso con la misma; y, finalmente, (6) el formato convencional tiene una mayor capacidad para generar intencionalidad de compra. Partiendo de estos resultados, debemos rechazar las hipótesis $\mathrm{H} 1 \mathrm{~b}$ y H1c, ya que la Telepromoción ha gustado menos, se considera menos atractiva, más molesta y genera menos intencionalidad de compra que el Spot. No obstante, la Telepromoción se considera más informativa que el Spot y con una diferencia notable. 
TABLA 5

Influencia del tipo de anuncio en las medidas afectivas de eficacia

\begin{tabular}{|l|c|c|c|c|c|c|}
\hline \multirow{2}{*}{$\begin{array}{c}\text { MEDIDAS AFECTIVAS Y } \\
\text { CONATIVAS }\end{array}$} & \multicolumn{2}{c|}{$\begin{array}{c}\text { Spot } \\
\text { CONia }\end{array}$} & \multicolumn{2}{c|}{ Telepromoción } & \multirow{2}{*}{$\boldsymbol{t}$} & \multirow{2}{*}{$\boldsymbol{p}$} \\
\cline { 2 - 5 } & Ded. & Media & D.T. & & \\
\hline Liking & 3.94 & 1.58 & 3.63 & 1.81 & 3.751 & 0.000 \\
\hline Atractivo & 3.57 & 1.39 & 3.43 & 1.42 & 1.982 & 0.048 \\
\hline Carácter informativo & 4.17 & 1.63 & 5.57 & 1.20 & -19.693 & 0.000 \\
\hline Credibilidad & 4.01 & 1.54 & 4.05 & 1.43 & -0.574 & 0.566 \\
\hline Grado de molestia & 3.89 & 1.24 & 4.75 & 1.31 & -13.378 & 0.000 \\
\hline Intención de compra & 3.86 & 2.15 & 3.18 & 2.01 & 6.639 & 0.000 \\
\hline
\end{tabular}

Al objeto de conocer con más detalle las diferencias existentes entre ambos formatos percibidas por los individuos, en la Tabla 6 se recogen los resultados de una análisis de diferencias de medias entre los diferentes ítems que se han utilizado para conformar el constructo de la actitud del anuncio. Como se puede observar, de los cinco ítems que integran la dimensión "atractivo" tan sólo en dos de ellos se observan diferencias significativas entre ambos tipos de formatos, percibiéndose el formato convencional como más atractivo y original. En cuanto a la dimensión "informativo", se observan diferencias significativas en los dos ítems que la integran, siendo el formato no convencional el que goza de un mayor carácter informativo de acuerdo con los individuos entrevistados. En la dimensión "molesto", al igual que en la dimensión anterior, los resultados reflejan la existencia de diferencias significativas en los tres ítems integrantes de dicha dimensión, siendo el formato no convencional el que se percibe como más molesto, destacando de forma notable el hecho de que este tipo de anuncios se confunde con la programación. Finalmente, en cuanto a los ítems de credibilidad no se aprecian diferencias entre ambos formatos.

Respecto a la interpretación de estos resultados, si bien algunos de los trabajos referenciados consideran el funcionamiento de los sistemas cognitivo y afectivo como sistemas independientes (TomKINS, 1970), otras fuentes reconocen que hay una alta interdependencia (Peter y Olson, 2005).

En este contexto existen fuentes que argumentan que actitudes positivas hacia los mensajes comerciales condicionan una mayor eficacia en términos de recuerdo (LARDINOIT, 1998; HACKLEY ET AL., 2005); sin embargo, como se ha señalado en el estado de la cuestión, en trabajos centrados en el Product placement (BALASUBRAMANIAN, 1994), el recuerdo (componente cognoscitiva) y las actitudes (componente afectiva) reportan niveles de eficacia de sentido contrario: el mayor recuerdo del Product placement se origina cuando las audiencias lo rechazan (atributos negativos) al ser identificado como un mensaje persuasivo. Este rechazo es más acusado cuando la ejecución formal del mismo hace que se explicite (mediante su identificación) su intencionalidad comercial a las audiencias; por ello, se genera un mayor recuerdo. Aunque el formato de estudio en este trabajo, la Telepromoción externa, está formalmente identificado como mensaje publicitario, y a priori no cabe inferir un posible rechazo derivado de la ocultación de sus objetivos, se puede concluir, tal y como muestran los resultados, que las asociaciones negativas que la Telepromoción genera son asociaciones explicitas de rechazo a un mensaje comercial por aparecer en un entorno y forma que se considera no es apropiado para un mensaje comercial: 
interrumpe la programación, es engañosa al percibirse disfrazada y confundirse con ella, resultando por ello más molesta y en general gusta menos. Otra posible interpretación de los resultados alcanzados, se puede encontrar en los trabajos de Russell (2002) y MiLes (2009), en los que se pone de manifiesto que en los formatos no convencionales, el nivel de incongruencia de la marca anunciada con el entorno/programa en el que aparece puede generar rechazo en la audiencia, el cual en sí mismo, es el que genera un mayor recuerdo. En nuestro estudio, por lo tanto, si la Telepromoción genera atributos negativos y a su vez se produce un mayor recuerdo en las audiencias, esto puede ser debido a la integración inadecuada de la marca anunciada con los contenidos del programa. Así mismo, otra posible argumentación de estos resultados inesperados se recoge en los trabajos de Singh y Cole (1993), StAnton y BuRKe (1998) y REINARES (2010), en los que se pone de manifiesto que las audiencias valoran de forma positiva los mensajes cortos y de esta manera aminoran su rechazo.

TABLA 6

Influencia del tipo de anuncio en los ítems del constructo "actitud hacia el anuncio"

\begin{tabular}{|c|c|c|c|c|c|c|c|}
\hline \multirow{2}{*}{\multicolumn{2}{|c|}{ MEDIDAS AFECTIVAS }} & \multicolumn{2}{|c|}{ Spot } & \multicolumn{2}{|c|}{ Telepromoción } & \multirow{2}{*}{$t$} & \multirow{2}{*}{$p$} \\
\hline & & Media & D.T. & Media & D.T. & & \\
\hline \multirow{5}{*}{ Atractivo } & Es un mensaje atractivo & 3.96 & 1.74 & 3.70 & 1.79 & 2.890 & 0.004 \\
\hline & Es un mensaje original & 3.61 & 1.79 & 3.42 & 1.74 & 2.173 & 0.030 \\
\hline & Este mensaje capta mi atención & 3.60 & 1.93 & 3.50 & 1.84 & 1.038 & 0.299 \\
\hline & El objetivo de este mensaje es entretener & 2.75 & 1.63 & 2.81 & 1.73 & -0.617 & 0.537 \\
\hline & Es un mensaje fácil de recordar & 4.19 & 2.03 & 4.13 & 1.79 & 0.632 & 0.527 \\
\hline \multirow{2}{*}{ Creíble } & Es un mensaje creíble & 4.27 & 1.77 & 4.38 & 1.68 & -1.234 & 0.218 \\
\hline & Este tipo de mensajes resultan muy convincentes & 3.73 & 1.70 & 3.70 & 1.64 & 0.321 & 0.748 \\
\hline \multirow{2}{*}{ Informativo } & Es un mensaje informativo & 4.09 & 1.82 & 5.50 & 1.37 & -17.626 & 0.000 \\
\hline & El objetivo de este mensaje es proporcionar información & 4.26 & 1.81 & 5.65 & 1.35 & -17.606 & 0.000 \\
\hline \multirow{3}{*}{ Molesto } & Este tipo de mensajes resultan engañosos & 3.83 & 1.89 & 4.26 & 1.84 & -4.611 & 0.000 \\
\hline & No me gusta que interrumpan el programa con este tipo de mensajes & 5.64 & 1.92 & 5.85 & 1.80 & -2.280 & 0.023 \\
\hline & Este mensaje se confunde con el programa en el que aparece & 1.62 & 1.27 & 4.60 & 2.27 & -32.604 & 0.000 \\
\hline
\end{tabular}

\subsection{Factores que influyen en la eficacia publicitaria}

En este apartado analizaremos la influencia que ejerce una serie de variables en la eficacia de la publicidad en los tres niveles señalados. A pesar de recoger su influencia en hipótesis generales, éstas se han contrastado a su vez para los dos formatos analizados de forma independiente, al objeto de explorar si los resultados que se han obtenido en las evidencias empíricas analizadas en el estado de la cuestión, que se corresponden en su mayoría con anuncios convencionales, se pueden extender también a la publicidad no convencional.

\section{Género}

En lo que a la variable género se refiere, los resultados de los análisis realizados se recogen en las Tablas 7 y 8 , de las que se desprende que el género (1) no influye en las medidas cognoscitivas de la eficacia de la publicidad en ninguno de los dos formatos analizados, salvo en el recuerdo sugerido de la marca para el formato 
convencional; (2) influye en las medidas afectivas, siendo las mujeres las que puntúan más alto en la mayoría de las medidas y además en ambos formatos, y, finalmente, (3) influyen en la intencionalidad de compra, siendo las mujeres también las que presentan unos niveles más altos en ambos formatos. Sobre la base de estos resultados, se puede extraer como conclusión que se acepta de forma parcial la hipótesis H2, ya que no se ha podido demostrar la influencia del género en las medidas cognoscitivas.
A pesar de que los resultados obtenidos puedan parecer contrarios a las fuentes que han demostrado la influencia de la variable género en la eficacia publicitaria (HEETER Y GREENBERG, 1985; SMITH, 1993; GUPTA Y GOULD, 1997), debe recordarse, como se señaló en el estado de la cuestión, que estas fuentes sólo demuestran influencias en la componente afectiva. Realmente no están contrastadas diferencias en el aprendizaje de los mensajes publicitarios entre hombres y mujeres.

TABLA 7

Influencia del género en las medidas cognoscitivas de eficacia

\begin{tabular}{|c|c|c|c|c|c|c|c|c|c|c|c|}
\hline \multirow{3}{*}{ MEDIDAS COGNOSCITIVAS } & \multirow{3}{*}{ CATEGORÍA } & \multicolumn{5}{|c|}{ Spot } & \multicolumn{5}{|c|}{ Telepromoción } \\
\hline & & \multicolumn{2}{|c|}{ Hombre } & \multicolumn{2}{|c|}{ Mujer } & \multirow{2}{*}{$\begin{array}{l}\chi^{2} \\
(p)\end{array}$} & \multicolumn{2}{|c|}{ Hombre } & \multicolumn{2}{|c|}{ Mujer } & \multirow{2}{*}{$\begin{array}{l}\chi^{2} \\
(p)\end{array}$} \\
\hline & & $\mathbf{N}$ & $\%$ & $\mathbf{N}$ & $\%$ & & $\mathbf{N}$ & $\%$ & $\mathbf{N}$ & $\%$ & \\
\hline \multirow{2}{*}{ Recuerdo espontáneo de la categoría de producto } & SÍ & 26 & 8.2 & 54 & 10.9 & \multirow{2}{*}{$\begin{array}{c}1.619 \\
(0.203)\end{array}$} & 100 & 31.3 & 168 & 33.8 & \multirow{2}{*}{$\begin{array}{c}0.531 \\
(0.466)\end{array}$} \\
\hline & NO & 293 & 91.8 & 443 & 89.1 & & 219 & 68.7 & 329 & 66.2 & \\
\hline \multirow{2}{*}{ Recuerdo espontáneo de la marca } & Sí & 20 & 6.3 & 43 & 8.7 & \multirow{2}{*}{$\begin{array}{c}1.548 \\
(0.213)\end{array}$} & 75 & 23.5 & 124 & 24.9 & \multirow{2}{*}{$\begin{array}{c}0.218 \\
(0.640)\end{array}$} \\
\hline & NO & 299 & 93.7 & 454 & 91.3 & & 244 & 76.5 & 373 & 75.1 & \\
\hline \multirow{2}{*}{$\begin{array}{l}\text { Recuerdo espontáneo de la categoría de producto } \\
\text { y de la marca }\end{array}$} & Sí & 20 & 6.3 & 42 & 8.5 & \multirow{2}{*}{$\begin{array}{c}1.317 \\
(0.251)\end{array}$} & 75 & 23.5 & 123 & 24.7 & \multirow{2}{*}{$\begin{array}{r}0.162 \\
(0.687)\end{array}$} \\
\hline & NO & 299 & 93.7 & 455 & 91.5 & & 244 & 76.5 & 374 & 75.3 & \\
\hline \multirow{2}{*}{ Recuerdo sugerido de la marca } & Sí & 32 & 10.7 & 78 & 17.1 & \multirow{2}{*}{$\begin{array}{c}6.006 \\
(0.014)\end{array}$} & 82 & 33.6 & 138 & 36.9 & \multirow{2}{*}{$\begin{array}{r}0.698 \\
(0.403)\end{array}$} \\
\hline & NO & 267 & 89.3 & 377 & 82.9 & & 162 & 66.4 & 236 & 63.1 & \\
\hline \multirow{2}{*}{ Reconocimiento del anuncio } & Sí & 117 & 43.8 & 169 & 44.8 & \multirow{2}{*}{$\begin{array}{c}0.064 \\
(0.800)\end{array}$} & 105 & 64.8 & 158 & 66.9 & \multirow{2}{*}{$\begin{array}{c}0.195 \\
(0.659)\end{array}$} \\
\hline & NO & 150 & 56.2 & 208 & 55.2 & & 57 & 35.2 & 78 & 33.1 & \\
\hline
\end{tabular}

TABLA 8

Influencia del género en las medidas afectivas y conativas de eficacia

\begin{tabular}{|c|c|c|c|c|c|c|c|c|c|c|c|c|}
\hline \multirow{3}{*}{$\begin{array}{l}\text { MEDIDAS AFECTIVAS Y } \\
\text { CONATIVAS }\end{array}$} & \multicolumn{6}{|c|}{ Spot } & \multicolumn{6}{|c|}{ Telepromoción } \\
\hline & \multicolumn{2}{|c|}{ Hombre } & \multicolumn{2}{|c|}{ Mujer } & \multirow{2}{*}{$t$} & \multirow{2}{*}{$p$} & \multicolumn{2}{|c|}{ Hombre } & \multicolumn{2}{|c|}{ Mujer } & \multirow{2}{*}{$T$} & \multirow{2}{*}{$p$} \\
\hline & Media & D.T. & Media & D.T. & & & Media & D.T. & Media & D.T. & & \\
\hline Liking & 3.68 & 1.55 & 4.12 & 1.57 & 3.854 & 0.000 & 3.36 & 1.77 & 3.81 & 1.81 & 3.461 & 0.001 \\
\hline Atractivo & 3.33 & 1.35 & 3.72 & 1.40 & 3.900 & 0.000 & 3.25 & 1.42 & 3.54 & 1.42 & 2.890 & 0.004 \\
\hline Carácter informativo & 3.90 & 1.62 & 4.34 & 1.62 & 3.728 & 0.000 & 5.44 & 1.23 & 5.65 & 1.18 & 2.384 & 0.017 \\
\hline Credibilidad & 3.67 & 1.52 & 4.24 & 1.51 & 5.208 & 0.000 & 3.86 & 1.49 & 4.18 & 1.38 & 3.092 & 0.002 \\
\hline Grado de molestia & 3.92 & 1.22 & 3.88 & 1.26 & -0.413 & 0.680 & 4.77 & 1.30 & 4.73 & 1.31 & -0.437 & 0.663 \\
\hline Intención de compra & 3.47 & 2.09 & 4.12 & 2.15 & 4.236 & 0.000 & 2.94 & 1.90 & 3.33 & 2.07 & 2.686 & 0.007 \\
\hline
\end{tabular}




\section{Edad}

Antes de proceder a analizar la influencia de la edad en la eficacia publicitaria, se ha procedido a reagrupar a los individuos en cuatro categorías: Adolescentes (entre 10 y 14 años), que representan el 4.6\%; jóvenes (entre 15 y 24 años), que suponen un $29.6 \%$ de la muestra; adultos (entre 25 y 54 años), que se corresponden con el $48.2 \%$ de los encuestados, y finalmente, los mayores (mayores de 55 años), que suponen el $17.4 \%$.

De los resultados de la Tabla 9, se puede extraer como conclusión que la edad, como era de esperar, es una variable que afecta a las medidas cognoscitivas de eficacia en ambos formatos, cuyos niveles se van reduciendo a medida que se incrementa la edad. No obstante, a nivel afectivo se aprecia que la edad tan sólo influye para ambos formatos en el liking y en la dimensión atractivo de la actitud hacia el anuncio, siendo los adolescentes y los mayores los que registran unas mayores puntuaciones. Finalmente, en cuanto a la intencionalidad, se observa cómo la edad no ejerce influencia alguna en ninguno de los dos formatos considerados. Estos resultados nos llevan a aceptar muy parcialmente la hipótesis $\mathrm{H} 3$.

TABLA 9

Influencia de la edad en las medidas cognoscitivas de eficacia

\begin{tabular}{|c|c|c|c|c|c|c|c|c|c|c|c|}
\hline \multirow{3}{*}{ MEDIDAS COGNOSCITIVAS } & \multirow{3}{*}{ CATEGORÍA } & \multicolumn{5}{|c|}{ Spot } & \multicolumn{5}{|c|}{ Telepromoción } \\
\hline & & ADOL. & JOV. & ADUL. & MAY. & & ADOL. & JOV. & ADUL. & MAY. & \\
\hline & & $\begin{array}{l}\mathbf{N} \\
\%\end{array}$ & $\begin{array}{l}\mathbf{N} \\
\%\end{array}$ & $\begin{array}{l}\mathbf{N} \\
\%\end{array}$ & $\begin{array}{l}\mathbf{N} \\
\%\end{array}$ & $(p)$ & $\begin{array}{l}\mathbf{N} \\
\%\end{array}$ & $\begin{array}{l}\mathbf{N} \\
\%\end{array}$ & $\begin{array}{l}\mathbf{N} \\
\%\end{array}$ & $\begin{array}{l}\mathbf{N} \\
\%\end{array}$ & $(p)$ \\
\hline \multirow{2}{*}{$\begin{array}{l}\text { Recuerdo espontáneo de la categoría de } \\
\text { producto }\end{array}$} & SÍ & $\begin{array}{c}9 \\
23.7\end{array}$ & $\begin{array}{c}28 \\
11.6\end{array}$ & $\begin{array}{l}30 \\
7.6\end{array}$ & $\begin{array}{c}13 \\
9.2\end{array}$ & \multirow{2}{*}{$\begin{array}{l}11.337 \\
(0.010)\end{array}$} & $\begin{array}{c}19 \\
50.0\end{array}$ & \begin{tabular}{|c}
85 \\
35.1
\end{tabular} & $\begin{array}{c}134 \\
34.0\end{array}$ & $\begin{array}{c}30 \\
21.1\end{array}$ & \multirow{2}{*}{$\begin{array}{l}14.723 \\
(0.002)\end{array}$} \\
\hline & NO & $\begin{array}{c}29 \\
76.3\end{array}$ & $\begin{array}{l}214 \\
88.4\end{array}$ & $\begin{array}{l}364 \\
92.4\end{array}$ & $\begin{array}{l}129 \\
90.8\end{array}$ & & $\begin{array}{c}19 \\
50.0\end{array}$ & $\begin{array}{l}157 \\
64.9\end{array}$ & $\begin{array}{l}260 \\
66.0\end{array}$ & $\begin{array}{l}112 \\
78.9\end{array}$ & \\
\hline \multirow{2}{*}{ Recuerdo espontáneo de la marca } & SÍ & $\begin{array}{c}8 \\
21.1\end{array}$ & $\begin{array}{c}26 \\
10.7\end{array}$ & $\begin{array}{l}24 \\
6.1\end{array}$ & $\begin{array}{c}5 \\
3.5\end{array}$ & \multirow{2}{*}{$\begin{array}{l}17.568 \\
(0.001)\end{array}$} & $\begin{array}{c}13 \\
34.2\end{array}$ & $\begin{array}{c}70 \\
28.9\end{array}$ & $\begin{array}{c}93 \\
23.6\end{array}$ & $\begin{array}{c}23 \\
16.2\end{array}$ & \multirow{2}{*}{$\begin{array}{c}9.988 \\
(0.019)\end{array}$} \\
\hline & NO & $\begin{array}{c}30 \\
78.9\end{array}$ & $\begin{array}{l}216 \\
89.3\end{array}$ & $\begin{array}{l}370 \\
93.9\end{array}$ & $\begin{array}{l}137 \\
96.5\end{array}$ & & $\begin{array}{c}25 \\
65.8\end{array}$ & \begin{tabular}{|l|}
172 \\
71.1
\end{tabular} & $\begin{array}{l}301 \\
76.4\end{array}$ & $\begin{array}{l}119 \\
83.8\end{array}$ & \\
\hline \multirow{2}{*}{$\begin{array}{l}\text { Recuerdo espontáneo de la categoría de } \\
\text { producto y de la marca }\end{array}$} & SÍ & $\begin{array}{c}8 \\
21.1\end{array}$ & $\begin{array}{c}26 \\
10.7\end{array}$ & $\begin{array}{l}23 \\
5.8\end{array}$ & $\begin{array}{c}5 \\
3.5\end{array}$ & \multirow{2}{*}{$\begin{array}{l}18.310 \\
(0.000)\end{array}$} & $\begin{array}{c}13 \\
34.2\end{array}$ & $\begin{array}{c}69 \\
28.5\end{array}$ & $\begin{array}{c}93 \\
23.6\end{array}$ & $\begin{array}{c}23 \\
16.2\end{array}$ & \multirow{2}{*}{$\begin{array}{c}9.544 \\
(0.023)\end{array}$} \\
\hline & NO & $\begin{array}{c}30 \\
78.9\end{array}$ & $\begin{array}{r}216 \\
89.3\end{array}$ & $\begin{array}{r}371 \\
94.2\end{array}$ & $\begin{array}{r}137 \\
96.5\end{array}$ & & $\begin{array}{c}25 \\
65.8 \\
\end{array}$ & \begin{tabular}{|c|}
173 \\
71.5
\end{tabular} & $\begin{array}{l}301 \\
76.4\end{array}$ & $\begin{array}{l}119 \\
83.8\end{array}$ & \\
\hline \multirow{2}{*}{ Recuerdo sugerido de la marca } & SÍ & $\begin{array}{c}4 \\
13.3 \\
\end{array}$ & $\begin{array}{c}44 \\
20.4 \\
\end{array}$ & $\begin{array}{c}53 \\
14.3 \\
\end{array}$ & $\begin{array}{c}9 \\
6.6 \\
\end{array}$ & \multirow{2}{*}{$\begin{array}{l}12.931 \\
(0.005)\end{array}$} & $\begin{array}{c}4 \\
16.0 \\
\end{array}$ & \begin{tabular}{|c|}
73 \\
42.2 \\
\end{tabular} & $\begin{array}{c}108 \\
35.9 \\
\end{array}$ & $\begin{array}{c}34 \\
28.6 \\
\end{array}$ & \multirow{2}{*}{$\begin{array}{l}10.061 \\
(0.018)\end{array}$} \\
\hline & NO & $\begin{array}{c}26 \\
86.7\end{array}$ & $\begin{array}{c}172 \\
79.6\end{array}$ & $\begin{array}{l}318 \\
85.7\end{array}$ & $\begin{array}{c}128 \\
93.4\end{array}$ & & $\begin{array}{c}21 \\
84.0\end{array}$ & \begin{tabular}{|l|}
100 \\
57.8
\end{tabular} & $\begin{array}{l}193 \\
64.1\end{array}$ & $\begin{array}{c}85 \\
71.4\end{array}$ & \\
\hline \multirow{2}{*}{ Reconocimiento del anuncio } & SÍ & $\begin{array}{c}14 \\
53.8\end{array}$ & $\begin{array}{c}88 \\
51.2\end{array}$ & $\begin{array}{c}126 \\
39.6\end{array}$ & $\begin{array}{c}59 \\
46.1\end{array}$ & \multirow{2}{*}{$\begin{array}{c}7.203 \\
(0.066)\end{array}$} & $\begin{array}{c}16 \\
76.2\end{array}$ & $\begin{array}{c}62 \\
62.0\end{array}$ & $\begin{array}{l}140 \\
72.5\end{array}$ & $\begin{array}{c}46 \\
54.1\end{array}$ & \multirow{2}{*}{$\begin{array}{l}10.731 \\
(0.013)\end{array}$} \\
\hline & NO & $\begin{array}{c}12 \\
46.2\end{array}$ & $\begin{array}{c}84 \\
48.8\end{array}$ & $\begin{array}{l}192 \\
60.4\end{array}$ & $\begin{array}{c}69 \\
53.9\end{array}$ & & $\begin{array}{c}5 \\
23.8\end{array}$ & $\begin{array}{c}38 \\
38.0\end{array}$ & $\begin{array}{c}53 \\
27.5\end{array}$ & $\begin{array}{c}39 \\
45.9\end{array}$ & \\
\hline
\end{tabular}


TABLA 10

Influencia de la edad en las medidas afectivas y conativas de eficacia

\begin{tabular}{|c|c|c|c|c|c|c|c|c|c|c|}
\hline \multirow{3}{*}{$\begin{array}{l}\text { MEDIDAS AFECTIVAS Y } \\
\text { CONATIVAS }\end{array}$} & \multicolumn{5}{|c|}{ Spot } & \multicolumn{5}{|c|}{ Telepromoción } \\
\hline & ADOL. & JOV. & ADUL. & MAY. & \multirow{2}{*}{$\begin{array}{c}F \\
(p)\end{array}$} & ADOL. & JOV. & ADUL. & MAY. & \multirow{2}{*}{$\begin{array}{c}F \\
(p)\end{array}$} \\
\hline & $\begin{array}{l}\text { Media } \\
\text { (D.T.) }\end{array}$ & $\begin{array}{l}\text { Media } \\
\text { (D.T.) }\end{array}$ & $\begin{array}{l}\text { Media } \\
\text { (D.T.) }\end{array}$ & $\begin{array}{l}\text { Media } \\
\text { (D.T.) }\end{array}$ & & $\begin{array}{l}\text { Media } \\
\text { (D.T.) }\end{array}$ & $\begin{array}{l}\text { Media } \\
\text { (D.T.) }\end{array}$ & $\begin{array}{l}\text { Media } \\
\text { (D.T.) }\end{array}$ & $\begin{array}{l}\text { Media } \\
\text { (D.T.) }\end{array}$ & \\
\hline Liking & $\begin{array}{c}4.58 \\
(1.46)\end{array}$ & $\begin{array}{c}3.81 \\
(1.52)\end{array}$ & $\begin{array}{c}3.87 \\
(1.60)\end{array}$ & $\begin{array}{c}4.18 \\
(1.59)\end{array}$ & $\begin{array}{c}4.008 \\
(0.008)\end{array}$ & $\begin{array}{c}4.27 \\
(1.69)\end{array}$ & $\begin{array}{c}3.00 \\
(1.69)\end{array}$ & $\begin{array}{c}3.78 \\
(1.78)\end{array}$ & $\begin{array}{c}4.10 \\
(1.82)\end{array}$ & $\begin{array}{l}16.226 \\
(0.000)\end{array}$ \\
\hline Atractivo & $\begin{array}{c}4.03 \\
(1.23)\end{array}$ & $\begin{array}{c}3.57 \\
(1.34)\end{array}$ & $\begin{array}{c}3.42 \\
(1.41)\end{array}$ & $\begin{array}{c}3.86 \\
(1.44)\end{array}$ & $\begin{array}{c}5.077 \\
(0.002)\end{array}$ & $\begin{array}{c}4.01 \\
(1.33)\end{array}$ & $\begin{array}{c}3.06 \\
(1.28)\end{array}$ & $\begin{array}{c}3.47 \\
(1.44)\end{array}$ & $\begin{array}{c}3.78 \\
(1.51)\end{array}$ & $\begin{array}{l}10.577 \\
(0.000)\end{array}$ \\
\hline Carácter informativo & $\begin{array}{c}4.17 \\
(1.55) \\
\end{array}$ & $\begin{array}{c}4.05 \\
(1.54) \\
\end{array}$ & $\begin{array}{c}4.13 \\
(1.67) \\
\end{array}$ & $\begin{array}{c}4.45 \\
(1.68) \\
\end{array}$ & $\begin{array}{c}1.938 \\
(0.122) \\
\end{array}$ & $\begin{array}{c}3.86 \\
(1.15) \\
\end{array}$ & $\begin{array}{c}5.72 \\
(1.12) \\
\end{array}$ & $\begin{array}{c}5.54 \\
(1.17) \\
\end{array}$ & $\begin{array}{c}5.32 \\
(1.38) \\
\end{array}$ & $\begin{array}{r}4.114 \\
(0.007) \\
\end{array}$ \\
\hline Credibilidad & $\begin{array}{c}4.27 \\
(1.39)\end{array}$ & $\begin{array}{c}4.08 \\
(1.44)\end{array}$ & $\begin{array}{c}3.88 \\
(1.58)\end{array}$ & $\begin{array}{c}4.18 \\
(1.59)\end{array}$ & $\begin{array}{c}2.011 \\
(0.111)\end{array}$ & $\begin{array}{c}4.46 \\
(1.15)\end{array}$ & $\begin{array}{c}4.01 \\
(1.29)\end{array}$ & $\begin{array}{c}4.06 \\
(1.49)\end{array}$ & $\begin{array}{c}4.01 \\
(1.55)\end{array}$ & $\begin{array}{c}1.109 \\
(0.345)\end{array}$ \\
\hline Grado de molestia & $\begin{array}{c}3.51 \\
(1.24)\end{array}$ & $\begin{array}{c}3.85 \\
(1.26) \\
\end{array}$ & $\begin{array}{c}3.95 \\
(1.27)\end{array}$ & $\begin{array}{c}3.97 \\
(1.12) \\
\end{array}$ & $\begin{array}{c}1.684 \\
(0.169) \\
\end{array}$ & $\begin{array}{c}4.29 \\
(1.16) \\
\end{array}$ & $\begin{array}{c}4.75 \\
(1.29) \\
\end{array}$ & $\begin{array}{c}4.80 \\
(1.34)\end{array}$ & $\begin{array}{c}4.76 \\
(1.25) \\
\end{array}$ & $\begin{array}{r}1.714 \\
(0.163) \\
\end{array}$ \\
\hline Intención de compra & $\begin{array}{c}4.00 \\
(2.23)\end{array}$ & $\begin{array}{c}3.84 \\
(2.17)\end{array}$ & $\begin{array}{c}3.88 \\
(2.14)\end{array}$ & $\begin{array}{c}3.82 \\
(2.13)\end{array}$ & $\begin{array}{c}0.095 \\
(0.963)\end{array}$ & $\begin{array}{c}3.30 \\
(2.23)\end{array}$ & $\begin{array}{c}3.17 \\
(1.98)\end{array}$ & $\begin{array}{c}3.13 \\
(1.99)\end{array}$ & $\begin{array}{c}3.27 \\
(2.04)\end{array}$ & $\begin{array}{c}0.207 \\
(0.892)\end{array}$ \\
\hline
\end{tabular}

\section{Clase social}

Antes de proceder a analizar la influencia de la clase social en la eficacia publicitaria, se ha recodificado la variable clase social en cuatro categorías, al objeto de que los estadísticos utilizados en los contrastes fuesen fiables. Como se observa en las Tablas 11 y 12, la clase social no afecta a las medidas cognitivas y conativas en ninguno de los dos formatos; mientras que en las medidas afectivas, en el liking, en el atractivo y en el carácter informativo, sí influ- ye, pero sólo en el formato no convencional, apreciándose que a medida que se incrementa el estatus social las puntuaciones de estas medidas disminuyen. Estos resultados son congruentes con el trabajo de DAHLÉN Y EDENIUS (2007), en el que se demuestra que la identificación del carácter persuasivo de los mensajes publicitarios, sean o no explícitos, por parte de niveles culturales altos condiciona mecanismos de protección en las audiencias ante dichos mensajes. Sobre esta base sólo es posible aceptar la hipótesis H4 de una forma muy parcial. 
TABLA 11

Influencia de la clase social en las medidas cognoscitivas de eficacia

\begin{tabular}{|c|c|c|c|c|c|c|c|c|c|c|c|}
\hline \multirow{3}{*}{$\begin{array}{c}\text { MEDIDAS } \\
\text { COGNOSCITIVAS }\end{array}$} & \multirow{3}{*}{ CATEGORÍA } & \multicolumn{5}{|c|}{ Spot } & \multicolumn{5}{|c|}{ Telepromoción } \\
\hline & & Alta & $\begin{array}{l}\text { Media- } \\
\text { Alta }\end{array}$ & $\begin{array}{l}\text { Media- } \\
\text { Media }\end{array}$ & $\begin{array}{l}\text { Media- } \\
\text { Baja/Baja }\end{array}$ & \multirow{2}{*}{$\begin{array}{l}\chi^{2} \\
(p)\end{array}$} & Alta & $\begin{array}{c}\text { Media- } \\
\text { Alta }\end{array}$ & $\begin{array}{l}\text { Media- } \\
\text { Media }\end{array}$ & $\begin{array}{l}\text { Media- } \\
\text { Baja/Baja }\end{array}$ & \multirow{2}{*}{$\begin{array}{l}\chi^{2} \\
(p)\end{array}$} \\
\hline & & $\begin{array}{l}\mathbf{N} \\
\%\end{array}$ & $\begin{array}{l}\mathbf{N} \\
\%\end{array}$ & $\begin{array}{l}\mathbf{N} \\
\%\end{array}$ & $\begin{array}{l}\mathbf{N} \\
\%\end{array}$ & & $\begin{array}{l}\mathbf{N} \\
\%\end{array}$ & $\begin{array}{l}\mathbf{N} \\
\%\end{array}$ & $\begin{array}{l}\mathbf{N} \\
\%\end{array}$ & $\begin{array}{l}\mathbf{N} \\
\%\end{array}$ & \\
\hline \multirow{2}{*}{$\begin{array}{l}\text { Recuerdo espontáneo de } \\
\text { la categoría de producto }\end{array}$} & SÍ & $\begin{array}{c}15 \\
13.4\end{array}$ & $\begin{array}{l}17 \\
9.4\end{array}$ & $\begin{array}{l}35 \\
9.8\end{array}$ & $\begin{array}{c}6 \\
7.9\end{array}$ & \multirow{2}{*}{$\begin{array}{c}1.877 \\
(0.598)\end{array}$} & $\begin{array}{c}39 \\
34.8\end{array}$ & $\begin{array}{c}65 \\
36.1\end{array}$ & $\begin{array}{c}113 \\
31.6\end{array}$ & $\begin{array}{c}24 \\
31.6\end{array}$ & \multirow{2}{*}{$\begin{array}{l}1.343 \\
(0.719)\end{array}$} \\
\hline & NO & $\begin{array}{c}97 \\
86.6\end{array}$ & $\begin{array}{c}163 \\
90.6\end{array}$ & $\begin{array}{l}323 \\
90.2\end{array}$ & $\begin{array}{c}70 \\
92.1\end{array}$ & & $\begin{array}{c}73 \\
65.2\end{array}$ & $\begin{array}{l}115 \\
63.9\end{array}$ & $\begin{array}{l}245 \\
68.4\end{array}$ & $\begin{array}{c}52 \\
68.4\end{array}$ & \\
\hline \multirow{2}{*}{$\begin{array}{l}\text { Recuerdo espontáneo de } \\
\text { la marca }\end{array}$} & SÍ & $\begin{array}{c}14 \\
12.5\end{array}$ & $\begin{array}{c}13 \\
7.2\end{array}$ & $\begin{array}{l}29 \\
8.1\end{array}$ & $\begin{array}{c}3 \\
3.9\end{array}$ & \multirow{2}{*}{$\begin{array}{c}4.845 \\
(0.184)\end{array}$} & $\begin{array}{c}29 \\
25.9\end{array}$ & $\begin{array}{c}46 \\
25.6\end{array}$ & $\begin{array}{c}82 \\
22.9\end{array}$ & $\begin{array}{c}19 \\
25.0\end{array}$ & \multirow{2}{*}{$\begin{array}{c}0.708 \\
(0.871)\end{array}$} \\
\hline & NO & $\begin{array}{c}98 \\
87.5\end{array}$ & $\begin{array}{c}167 \\
92.8\end{array}$ & $\begin{array}{l}329 \\
91.9\end{array}$ & $\begin{array}{c}73 \\
96.1\end{array}$ & & $\begin{array}{c}83 \\
74.1\end{array}$ & $\begin{array}{l}134 \\
74.4\end{array}$ & $\begin{array}{l}276 \\
77.1\end{array}$ & $\begin{array}{c}579 \\
75.0\end{array}$ & \\
\hline \multirow{2}{*}{$\begin{array}{l}\text { Recuerdo espontáneo de } \\
\text { la categoría de producto y } \\
\text { de la marca }\end{array}$} & SÍ & $\begin{array}{c}14 \\
12.5\end{array}$ & $\begin{array}{l}12 \\
6.7\end{array}$ & $\begin{array}{l}29 \\
8.1\end{array}$ & $\begin{array}{c}3 \\
3.9\end{array}$ & \multirow{2}{*}{$\begin{array}{c}5.224 \\
(0.156)\end{array}$} & $\begin{array}{c}29 \\
25.9\end{array}$ & $\begin{array}{c}46 \\
25.6\end{array}$ & $\begin{array}{c}81 \\
22.6\end{array}$ & $\begin{array}{c}19 \\
25.0\end{array}$ & \multirow{2}{*}{$\begin{array}{c}0.864 \\
(0.834)\end{array}$} \\
\hline & NO & $\begin{array}{c}98 \\
87.5\end{array}$ & $\begin{array}{l}168 \\
93.3\end{array}$ & $\begin{array}{l}329 \\
91.9\end{array}$ & $\begin{array}{c}73 \\
96.1\end{array}$ & & $\begin{array}{c}83 \\
74.1\end{array}$ & $\begin{array}{c}134 \\
74.4\end{array}$ & $\begin{array}{l}277 \\
77.4\end{array}$ & $\begin{array}{c}57 \\
75.0\end{array}$ & \\
\hline \multirow{2}{*}{$\begin{array}{l}\text { Recuerdo sugerido de la } \\
\text { marca }\end{array}$} & SÍ & $\begin{array}{c}16 \\
16.3\end{array}$ & $\begin{array}{c}30 \\
17.9\end{array}$ & $\begin{array}{c}43 \\
13.1\end{array}$ & $\begin{array}{c}10 \\
13.7\end{array}$ & \multirow{2}{*}{$\begin{array}{c}2.275 \\
(0.517)\end{array}$} & $\begin{array}{c}32 \\
38.6\end{array}$ & $\begin{array}{c}44 \\
32.8\end{array}$ & $\begin{array}{l}102 \\
36.8\end{array}$ & $\begin{array}{c}17 \\
29.8\end{array}$ & \multirow{2}{*}{$\begin{array}{l}1.767 \\
(0.622)\end{array}$} \\
\hline & NO & $\begin{array}{c}82 \\
83.7\end{array}$ & $\begin{array}{l}138 \\
82.1\end{array}$ & $\begin{array}{l}286 \\
86.9\end{array}$ & $\begin{array}{c}63 \\
86.3\end{array}$ & & $\begin{array}{c}51 \\
61.4\end{array}$ & $\begin{array}{c}90 \\
67.2\end{array}$ & $\begin{array}{l}175 \\
63.2\end{array}$ & $\begin{array}{c}40 \\
70.2\end{array}$ & \\
\hline \multirow{2}{*}{$\begin{array}{l}\text { Reconocimiento del } \\
\text { anuncio }\end{array}$} & SÍ & $\begin{array}{c}32 \\
39.0 \\
\end{array}$ & $\begin{array}{c}60 \\
43.5 \\
\end{array}$ & $\begin{array}{l}135 \\
47.2 \\
\end{array}$ & $\begin{array}{c}25 \\
39.7\end{array}$ & \multirow{2}{*}{$\begin{array}{c}2.484 \\
(0.478)\end{array}$} & $\begin{array}{c}29 \\
56.9\end{array}$ & $\begin{array}{c}63 \\
70.0 \\
\end{array}$ & $\begin{array}{c}127 \\
72.6 \\
\end{array}$ & $\begin{array}{c}20 \\
50.0\end{array}$ & \multirow{2}{*}{$\begin{array}{l}10.441 \\
(0.015)\end{array}$} \\
\hline & NO & $\begin{array}{c}50 \\
61.0\end{array}$ & $\begin{array}{c}78 \\
56.5\end{array}$ & $\begin{array}{c}151 \\
52.8\end{array}$ & $\begin{array}{c}38 \\
60.3\end{array}$ & & $\begin{array}{c}22 \\
43.1\end{array}$ & $\begin{array}{c}27 \\
30.0\end{array}$ & $\begin{array}{c}48 \\
27.4\end{array}$ & $\begin{array}{c}20 \\
50.0\end{array}$ & \\
\hline
\end{tabular}

TABLA 12

Influencia de la clase social en las medidas afectivas y conativas de eficacia

\begin{tabular}{|c|c|c|c|c|c|c|c|c|c|c|}
\hline \multirow{3}{*}{$\begin{array}{l}\text { MEDIDAS AFECTIVAS Y } \\
\text { CONATIVAS }\end{array}$} & \multicolumn{5}{|c|}{ Spot } & \multicolumn{5}{|c|}{ Telepromoción } \\
\hline & Alta & $\begin{array}{l}\text { Media- } \\
\text { Alta }\end{array}$ & $\begin{array}{l}\text { Media- } \\
\text { Media }\end{array}$ & $\begin{array}{c}\text { Media-Baja/ } \\
\text { Baja }\end{array}$ & \multirow{2}{*}{$\begin{array}{c}F \\
(p)\end{array}$} & Alta & $\begin{array}{l}\text { Media- } \\
\text { Alta }\end{array}$ & $\begin{array}{l}\text { Media- } \\
\text { Media }\end{array}$ & $\begin{array}{l}\text { Media-Baja/ } \\
\text { Baja }\end{array}$ & \multirow{2}{*}{$\begin{array}{c}F \\
(p)\end{array}$} \\
\hline & $\begin{array}{l}\text { Media } \\
\text { (D.T.) }\end{array}$ & $\begin{array}{c}\text { Media } \\
\text { (D.T.) }\end{array}$ & $\begin{array}{l}\text { Media } \\
\text { (D.T.) }\end{array}$ & $\begin{array}{l}\text { Media } \\
\text { (D.T.) }\end{array}$ & & $\begin{array}{l}\text { Media } \\
\text { (D.T.) }\end{array}$ & $\begin{array}{l}\text { Media } \\
\text { (D.T.) }\end{array}$ & $\begin{array}{c}\text { Media } \\
\text { (D.T.) }\end{array}$ & $\begin{array}{l}\text { Media } \\
\text { (D.T.) }\end{array}$ & \\
\hline Liking & $\begin{array}{c}4.19 \\
(1.65)\end{array}$ & $\begin{array}{c}3.83 \\
(1.59)\end{array}$ & $\begin{array}{c}3.89 \\
(1.51)\end{array}$ & $\begin{array}{c}4.03 \\
(1.72)\end{array}$ & $\begin{array}{c}1.422 \\
(0.235)\end{array}$ & $\begin{array}{c}3.18 \\
(1.73)\end{array}$ & $\begin{array}{c}3.36 \\
(1.82)\end{array}$ & $\begin{array}{c}3.80 \\
(1.74)\end{array}$ & $\begin{array}{c}3.99 \\
(1.90)\end{array}$ & $\begin{array}{c}5.801 \\
(0.001)\end{array}$ \\
\hline Atractivo & $\begin{array}{c}3.81 \\
(1.54)\end{array}$ & $\begin{array}{c}3.39 \\
(1.33)\end{array}$ & $\begin{array}{c}3.53 \\
(1.36)\end{array}$ & $\begin{array}{c}3.64 \\
(1.49)\end{array}$ & $\begin{array}{c}2.253 \\
(0.081)\end{array}$ & $\begin{array}{c}3.13 \\
(1.26)\end{array}$ & $\begin{array}{c}3.27 \\
(1.45)\end{array}$ & $\begin{array}{c}3.46 \\
(1.40)\end{array}$ & $\begin{array}{c}3.79 \\
(1.45)\end{array}$ & $\begin{array}{c}3.969 \\
(0.008)\end{array}$ \\
\hline Carácter informativo & $\begin{array}{c}4.10 \\
(1.46)\end{array}$ & $\begin{array}{c}4.27 \\
(1.71)\end{array}$ & $\begin{array}{c}4.12 \\
(1.67)\end{array}$ & $\begin{array}{c}4.12 \\
(1.71)\end{array}$ & $\begin{array}{c}0.430 \\
(0.732) \\
\end{array}$ & $\begin{array}{c}5.22 \\
(1.22) \\
\end{array}$ & $\begin{array}{c}5.57 \\
(1.23) \\
\end{array}$ & $\begin{array}{c}5.68 \\
(1.12) \\
\end{array}$ & $\begin{array}{c}5.60 \\
(1.31) \\
\end{array}$ & $\begin{array}{c}4.268 \\
(0.005)\end{array}$ \\
\hline Credibilidad & $\begin{array}{c}4.04 \\
(1.48) \\
\end{array}$ & $\begin{array}{c}3.90 \\
(1.51) \\
\end{array}$ & $\begin{array}{c}4.05 \\
(1.58) \\
\end{array}$ & $\begin{array}{c}3.84 \\
(1.65) \\
\end{array}$ & $\begin{array}{c}0.710 \\
(0.546) \\
\end{array}$ & $\begin{array}{c}3.84 \\
(1.33) \\
\end{array}$ & $\begin{array}{c}3.95 \\
(1.47) \\
\end{array}$ & $\begin{array}{c}4.08 \\
(1.42) \\
\end{array}$ & $\begin{array}{c}4.29 \\
(1.53) \\
\end{array}$ & $\begin{array}{c}1.826 \\
(0.141) \\
\end{array}$ \\
\hline Grado de molestia & $\begin{array}{c}3.84 \\
(1.27) \\
\end{array}$ & $\begin{array}{c}3.90 \\
(1.23) \\
\end{array}$ & $\begin{array}{c}3.89 \\
(1.25) \\
\end{array}$ & $\begin{array}{c}3.82 \\
(1.30) \\
\end{array}$ & $\begin{array}{c}0.130 \\
(0.942) \\
\end{array}$ & $\begin{array}{c}4.76 \\
(1.23) \\
\end{array}$ & $\begin{array}{c}4.78 \\
(1.24) \\
\end{array}$ & $\begin{array}{c}4.74 \\
(1.33) \\
\end{array}$ & $\begin{array}{c}4.70 \\
(1.37) \\
\end{array}$ & $\begin{array}{c}0.076 \\
(0.973) \\
\end{array}$ \\
\hline Intención de compra & $\begin{array}{c}4.10 \\
(2.09)\end{array}$ & $\begin{array}{c}3.98 \\
(2.26)\end{array}$ & $\begin{array}{c}3.73 \\
(2.09)\end{array}$ & $\begin{array}{c}3.41 \\
(2.16)\end{array}$ & $\begin{array}{c}2.146 \\
(0.093)\end{array}$ & $\begin{array}{c}2.99 \\
(1.81)\end{array}$ & $\begin{array}{c}3.08 \\
(2.10)\end{array}$ & $\begin{array}{c}3.18 \\
(1.98)\end{array}$ & $\begin{array}{c}3.28 \\
(2.09)\end{array}$ & $\begin{array}{c}0.409 \\
(0.747)\end{array}$ \\
\hline
\end{tabular}




\section{CONCLUSIONES}

En la planificación publicitaria, una de las decisiones más importantes radica en la adecuada selección del formato para desarrollar el mensaje en función de los objetivos y su potencial eficacia. La literatura académica y profesional ha analizado ampliamente la eficacia de la publicidad en televisión de formatos convencionales como son los Spots. Sin embargo, por los motivos expuestos en este trabajo, existe una escasez de estudios de eficacia sobre las denominadas formas no convencionales de publicidad. Esta carencia justifica la investigación de la eficacia del formato no convencional Telepromoción externa y su planteamiento de forma comparada con la publicidad convencional como se propone en este trabajo. De esta manera se han podido aportar las necesarias evidencias empíricas para su desarrollo teórico.

Tampoco se ha desarrollado suficientemente en este ámbito, posiblemente por la complejidad técnica de una investigación en entorno real, el análisis comparativo de eficacia de formatos convencionales (Spot) frente a los no convencionales en televisión como el que se plantea en esta investigación.

En el presente trabajo hemos aplicado un procedimiento de investigación, que ha permitido integrar todas aquellas variables y niveles que de forma fragmentada se aplican en la investigación académica sobre eficacia publicitaria en las formas convencionales. Asimismo, se cumple con los objetivos de contrastar en un entorno real la eficacia comparada de una Telepromoción frente a los Spots y aportar evidencias empíricas necesarias en el ámbito académico que justifican adecuadamente su aplicación en la práctica empresarial. PIETERS Y Bijmolt (1997) argumentan la necesidad de considerar únicamente válidos, los trabajos de investigación aplicados en un entorno real. Según exponen estos autores, con respecto a este objeto de estudio, no se pueden hacer generalizaciones sobre datos que no hayan sido obtenidos en un entorno real y a partir de muestras de estudiantes. Ésta es una afirmación necesariamente compartida como demuestra la metodología que se propone en este trabajo.

También desde un punto de vista metodológico, para cuantificar la componente cognitiva se han utilizado medidas de recuerdo espontáneo, sugerido y verificado de producto, marca $\mathrm{y}$ anuncio, y medidas de reconocimiento del anuncio. Diferentes trabajos reportan la necesidad de utilizar simultáneamente el recuerdo espontáneo y sugerido para medir el efecto cognitivo de la publicidad (MOORMAN ET AL., 2007). De Pelsmacker et AL. ( 2005b) señalan cómo el recuerdo espontáneo es una medida más fuerte de la memoria y por ello más difícil de obtener frente al recuerdo sugerido. En este sentido es frecuente encontrar, especialmente en la praxis de los institutos de investigación, trabajos que sólo presentan cifras de recuerdo asistido y/o reconocimiento, dado los bajísimos niveles que genera el recuerdo publicitario espontáneo en trabajos desarrollados en un entorno real. Sin embargo, el diseño de esta investigación permite trabajar con ambos tipos de medidas en la línea de trabajos como los de Pieters y Bijmolt (1997). El estudio simultáneo del recuerdo espontáneo y sugerido permite determinar las relaciones entre ambos. Dadas las características del recuerdo espontáneo se puede inferir la especial capacidad de las formas no convencionales para actuar sobre una componente más compleja del recuerdo.

Como se ha señalado, la Telepromoción externa ha obtenido niveles de recuerdo muy elevados en relación a la publicidad convencional. Se trata de un formato no convencional muy reconocido por los anunciantes para proporcionar más información sobre la marca que un anuncio convencional (SMIT ET AL., 2009), y que como se ha contrastado en este estudio, efectivamente es percibido por la audiencia como un formato más informativo que el Spot. Sin embargo, también se ha contrastado como la Telepromoción proporciona una calidad de recuerdo inferior a la aportada por el Spot. Esto 
evidencia que, a pesar de la valoración de los agentes publicitarios del potencial de comunicación de la Telepromoción, se trata de un formato que en efecto proporciona mayor notoriedad, pero no traslada los contenidos a las audiencias, dado que no son capaces de recordar aspectos del contenido de la Telepromoción al mismo nivel que en los Spots. Por ello resultaría especialmente indicado para anunciantes nuevos o poco conocidos, y/o con presupuestos modestos, y que necesiten notoriedad a corto plazo; mientras que el Spot seguirá siendo una forma eficaz en la comunicación para anunciantes que compiten en categorías de producto en mercados maduros, donde es importante que las audiencias comprendan los atributos diferenciales de cada marca.

Dado que son numerosos los trabajos que, con metodologías experimentales y desarrollos cualitativos, han valorado la componente afectiva de los formatos no convencionales, ha resultado de gran importancia desarrollar un trabajo de carácter cuantitativo cuyos resultados permiten una mayor representatividad que la habitual para esta componente de la eficacia.

Por otra parte, un valor añadido de esta línea de investigación ha sido entender las relaciones entre eficacia cognoscitiva y eficacia afectiva. Partiendo de este objetivo, una de las principales aportaciones de este estudio ha sido la aplicación de la investigación en un entorno real y de forma simultanea, para contrastar tanto la eficacia cognoscitiva como la eficacia afectiva a partir de la misma muestra y utilizando los mismos formatos de estudio.

Los resultados de nuestro estudio han mostrado que, si una FNC como la Telepromoción externa genera un mayor recuerdo, el mismo puede estar directamente relacionado con una valoración negativa de la misma por parte del público objetivo. La eficacia en términos afectivos funciona en un sentido diferente que la eficacia en términos cognoscitivos. A pesar de los elevados niveles de recuerdo que genera la Telepromoción frente al Spot, en el ámbito afectivo y conativo, gusta menos y resulta más molesta, además de generar una menor intencionalidad de compra.

Estos resultados sobre un nuevo formato de estudio son otro indicador, en la línea del trabajo de BALASUbramanian (1994) sobre Product Placement, de que las FNC actúan sobre las actitudes de las audiencias de una forma diferente a la publicidad convencional y, que por lo tanto, es necesario realizar nuevos estudios para contrastar las relaciones entre las diferentes variables de eficacia. Ello trae consigo evidentes implicaciones en la gestión de las FNC, afectando, en especial, a los objetivos de comunicación de las campañas que utilizan estos formatos.

Respecto a las hipótesis relacionadas con la influencia sobre la eficacia publicitaria de las variables sociodemográficas, los resultados indican que existe una influencia similar para ambos formatos y coherente con los resultados recogidos en la literatura. Estas hipótesis básicas resultaban fundamentales para aportar evidencias empíricas sobre la capacidad discriminante en la variable formato no convencional con respecto a su eficacia frente a la publicidad al uso, además de permitir actuar sobre aquellas dimensiones de eficacia en las que puede existir una mayor capacidad en el formato no convencional a partir de una presión publicitaria inferior a la conseguida con los planes de medios basados en publicidad convencional.

Por otra parte, proporcionar conocimiento sobre la influencia de las variables sociodemográficas, potencia la optimización de una planificación cualitativa del medio, junto con la necesidad de ajustar los elementos creativos y los objetivos publicitarios en función de las variables que definen al público objetivo de la campaña. Por ejemplo, de acuerdo a los resultados de este estudio, en el caso de la variable género, se conseguiría mejorar la eficacia de la componente afectiva y conativa, en las campañas donde por tipología de producto esto se considera necesario. Sin embargo, respecto a la edad, sería conveniente actuar sobre la componente cognoscitiva. Esta contrastación es espe- 
cialmente relevante en la medida en que los hábitos de audiencia varían, con respecto al tiempo de exposición diario al medio televisión, entre los diferentes grupos de edad ${ }^{7}$. También es preciso considerar que la fragmentación de audiencias dificulta desarrollar planes de medios con frecuencias medias de impacto elevadas en determinados rangos de edad (REINARES Y REINARES, 2008). En cuanto a la clase social, al igual que en la variable género, la utilización de estas formas condicionaría una posible segmentación del público objetivo respecto a la dimensión afectiva de la eficacia.

Por ultimo señalar que este trabajo ha tratado de acercar la investigación académica a las necesidades derivadas de la gestión de las actuales prácticas publicitarias en televisión, que posiblemente por su novedad y dinamismo aún no han sido suficientemente abordadas.

Respecto a las limitaciones de este trabajo de investigación, éstas se deben principalmente al necesario ajuste entre los objetivos y la metodología empleada. Gran parte de la literatura sobre formas no convencionales como el product placement, valoran como elementos moderadores de la eficacia, la implicación de la audiencia hacia el producto-marca contenido en dicha forma no convencional. Por ello debe considerarse como limitación la ausencia de control sobre las categorías de productos anunciados. Al tratarse de un entorno real nos encontrábamos condicionados a limitaciones impuestas por anunciantes y cadena, siendo nuestro principal requisito que, los productos y campañas fueran nuevas, y se iniciaran en el mismo bloque de estudio para que no hubiera interferencia de producto/campaña previa. Estos requisitos impidieron el control de otro tipo de variables como los productos anunciados. Por otra parte, dado que la duración de la Telepromoción y el Spot objeto de estudio, es inherente a los propios formatos, y por lo tanto no se puede controlar, asumimos de acuerdo a la literatura sobre la influencia de la duración del formato publicitario que, a priori, la diferente duración de ambos formatos, ha podido suponer una limitación en el estudio; sin embargo se ha procedido a considerar las referencias oportunas en la discusión de resultados sobre la influencia de esta variable en los mismos.

Por último, como se puede observar en la revisión teórica del estado de la cuestión, la literatura previa se ha centrado en un reducido número de tipologías de FNC y apenas existen estudios sobre el formato objeto de estudio de este trabajo, la Telepromoción externa; por otra parte, los estudios que plantean un análisis comparado entre un formato no convencional y la publicidad convencional también son escasos. Ambas carencias ha supuesto una limitación para el planteamiento de las hipótesis de trabajo en nuestra investigación.

Como futuras líneas de investigación, parece conveniente desarrollar a partir de la metodología propuesta en este trabajo, nuevos estudios para conocer las relaciones entre las diferentes variables de eficacia en el resto de formatos no convencionales. Por otra parte, dado que el ámbito geográfico de la investigación empírica sólo permite generalizar los resultados de los análisis a la población de la que procede la muestra, sería conveniente replicar esta investigación en otras zonas geográficas. En este contexto, además se propone extender este estudio a nivel europeo. Ya se ha justificado que la legislación propiciará un desarrollo homogéneo de estas prácticas publicitarias en países con un menor desarrollo en la actualidad. También se considera necesario, una vez se ha contrastado que la eficacia en términos afectivos funciona en un sentido diferente que la eficacia en términos cognoscitivos, profundizar en el origen de estos resultados y

\footnotetext{
7 En el trabajo de BusQueT ET AL. (2009), las diferencias del tiempo medio de audiencia en televisión pueden llegar a los 90 minutos diarios en función de la edad. La media diaria es de 3 horas 17 minutos y el perfil que menos televisión consume al día son los jóvenes entre 13 y 24 años, con una media de consumo de 2 horas 10 minutos.
} 
para cada uno de los niveles de respuesta. Finalmente, en la línea de la discusión de nuestros resultados, un paso obligado posterior se dirigirá hacia la identificación las variables creativas, de medios y relacionadas con la audiencia que pueden estar implicadas en los resultados exploratorios obtenidos en este trabajo.

\section{REFERENCIAS BIBLIOGRÁFICAS}

AAKer, D. A. y Bruzzone, D. E. (1981), "Viewer perceptions of prime-time television advertising", Journal of Advertising Research, 21(5), 15-23.

Amos, C., Holmes, G. y Strutton, D. (2008), "Exploring the relationship between celebrity endorser effects and advertising effectiveness A quantitative synthesis of effect size", International Journal of Advertising, 27(2), 209-234.

Arrazola, M., Hevia, J., Reinares, P. y ReINARES, R. (2010), "Do new forms of television advertising occasion better recall than traditional advertising spots?: an analysis proposal by means of probit models". En Proceedings of the European Advertising Academy Icoria, European Advertising Academy: Madrid.

Auty, S. Y LewIS, C. (2004), "Exploring children's choice: The reminder effect of product placement", Psychology and Marketing, 21(9), 217-244.

AVEry, R. J. y Ferraro, R. (2000), "Verisimilitude or advertising? Brand appearances on primetime television", The Journal of Consumer Affairs, 34 (2), 217-244.

Backstage (2008), Focus. Sponsoring TV: "Hoeveel is assez?. Marzo, disponible en: http://www.ipb.be/upload/TV/marketing/formats/sponsoringhoeveel/sponsoringhoeveel_fr.pdf, 15/10/2009.

BALASUBRAMANian, S. K. (1994), "Beyond Advertising and Publicity: Hybrid Messages and Public Policy Issues", Journal of Advertising, 23, (4), 29-46.
Bartel-Sheehan, K. y Guo, A. (2005), "Leaving on a branded jet plane: An exploration of audience attitudes towards product assimilation in television content", Journal of Current Issues and Research in Advertising, 27(1), 79-91.

Beerli Palacio, A. y Martín Santana, J.D. (1998), "Memory-Based Advertising Effectiveness Techniques: Recall versus Recognition". En Zopounidis, C. Y Pardalos, P.M. (Eds.), Managing in Uncertainty: Theory and Practice, Kluwer Academic Publishers: Amsterdam. 183-201.

Beerli Palacio, A. y Martín Santana, J.D. (1999), "Procedimiento de evaluación de la eficacia de anuncios publicitarios", Revista Española de Investigación de Marketing ESIC, 3(1), 37-62.

Bigné Alcañiz, E. y Miquel Romero, M. J. (1996), "La duración del anuncio: efectos cognitivos y afectivos". En LuQue, T. (Eds.), La empresa en una economía globalizada: retos y cambios. Vol. IB, Asociación Europea de Dirección y Economía de la Empresa: Granada. 767-778

Bijmolt, T. H. A., ClaAassen, W. y Brus, B. (1998), “Children's understanding of TV advertising: Effects of age, gender and parental influence", Journal of Consumer Policy, 21(2), 171-194.

BloXHAM, M. (1998), "Brand affinity and television programme sponsorship", International Journal of Advertising, 17(1), 89-98.

Brennan, I., Dubas, K. M. Y Babin, L. A. (1999), "The Influence of Product-Placement Type \& Exposure Time on ProductPlacement Recognition", International Journal of Advertising, 18(3), 323-337.

Busquet, J., Perales, A. Y Reinares, P. (2009), "La audiencia infantil de televisión en España: ni tan escasa ni tan uniforme", Telos: Cuadernos de comunicación e innovación, (81), 129-141.

CARAT (2005), Quantitative impact of the regulation on TV advertising markets in EU Member States, EEA countries, the new 
Member States and a number of third party countries Spain. Report 3 18/18. Stages B and $\mathrm{C}$, disponible en: http://ec.europa.eu/ avpolicy/docs/library/studies/2003/44_03_r e_us.pdf, 20/5/2009.

CARAt CRystal y Bird \& Bird (2002), Etude Sur Le Développement des Nouvelles Techniques Publicitaires. Rapport Final avril, France : Carat Crystal.

Chapman, P.S. Y Beltramini, R.F. (2000), "Infomercials revisited: perspectives of advertising professionals", Journal of advertising Research, 40(5), 24-31.

Chowdhury, R., Finn, A. y Olsen,G. (2007), "Investigating the Simultaneous Presentation of Advertising and Television Programming", Journal of Advertising , 36 (3), 8596.

Cowley, E. y Barron, C. (2008), "When Product Placement Goes Wrong: The Effects of Program Liking and Placement Prominence", Journal of Advertising, 37(1), pp. 89-98.

D’ Astous, A. y SÉGuin, N. (1999), “Consumer reactions to product placement strategies in television sponsorship", European Journal of Marketing, 33(9-10), 896-910.

DAHLÉN, M. Y EDENIUS, M. (2007), "When is advertising advertising? Comparing responses to traditional and non- traditional advertising media", Journal of Current Issues and Research in Advertising, 29(1), 33-42.

DARLEY, W.K. y SMith, R. E. (1995), “Gender Differences in Information Processing Strategies: An Empirical Test of the Selectivity in Advertising Response", Journal of Advertising, 24(1), 41-56.

Davies, G. Y Rojas-Mendez, J. I. (2005), "Avoiding Television Advertising: Some Explanations from Time Allocation Theory", Journal of Advertising Research, 45(1) 34-48.

Dawson, CH. (1996), "Television advertising: In need of reinvention?", International Journal of Advertising, 15(4), 302-313.

De Pelsmacker P. Geuens M. y Anckaert P. (2005a), "Advertising for New and Existing
Brands: The Impact of Media Context and Type of Advertisement", Journal of Marketing Communications, 11(2), 113-128.

De Pelsmacker P., Geuens M. y Anckaert, P. (2002), "Media context and advertising effectiveness: the role of context style, context quality and context-ad similarity", Journal of Advertising, 31(2), 49-61.

De Pelsmacker, P, Geuens, M. y Van Den Bergh, J. (2005b), Marketing Communicatie, Peason Education.

Droulers, O. (2006), "Influence de l'âge sur la mémorisation des marques présentées dans des publicités à la télévision", Revue Française du Marketing, (208), 49-59.

Dubow, J. S. (1994), "Point of view: Recall revisited: Recall redux", Journal of Advertising Research, 34(3), 92-99.

Edell, J. A. Y Burke, M. C. (1987), "The power of feelings in understanding advertising effects", Journal of Consumer Research, 14(3), 421-433.

EgTA (2008a), "Background - Special advertising formats". TV Focus. The European Data Base for television effectiveness, disponible en: http://tvfocus.egta.com/background/special_advertising_formats.php, 21/03/10.

Egta (2008b), Egta Practical guide on the EU directive on AMS, Brussels: Egta. Association of television and radio sales houses.

Elliott, M. T. Y LOCKARD, P. (1996), “An analysis of information content in infomercial programs", Journal of Direct Marketing, 10(2), 44-55.

Elpers, W., Josephine, L. C. M., Wedel, M. Y Pieters, R. G. M. (2003), "Why do consumers stop viewing television commercials? Two experiments on the influence of moment-to-moment entertainment and information value", Journal of Advertising Research, 40(4), 437-453.

European Commission (2007), "Presenting the new Audiovisual Media Services Without Frontiers Directive: MEMO/07/206", disponible en: http://europa.eu/rapid/pressRelea- 
sesAction.do? reference $=$ MEMO/07/206,10/ 05/2007.

FABIAN, G.S. (1986), "15-second commercials: The inevitable evolution", Journal of Advertising Research, 26(4), R C3-5.

FARRÉ, J. y FERNÁNDEZ, J. (2005), La publicidad no convencional en la televisión generalista, Quaderns del CAC, (22), 3-20.

Furnham, A., Bergland, J. y Gunter, B. (2002), "Memory for television advertisements as a function of advertisement-programme congruity", Applied Cognitive Psychology, 16(5), 525-545.

GÁZQUEZ ABAD, J.C., JIMÉNEZ CASTILLO, D., SÁEZ GONZÁLEZ, E. Y SÁNCHEZ PÉREZ, M. (2010), "El comportamiento de cambio de canal del telespectador en prime time. Análisis a través de un panel de audiometría", Revista Española de Investigación de Marketing ESIC, 14(2), 31-66.

Guido, G., Peluso, A.M., Tedeschi, P., Nicole, C., Lauretti, C. y Caciula, A. (2010), "Acceptance of Product Placement in Italy: Effects of Personality and Product/Consumer Interactions", International Journal of Marketing Studies, (2) 2, 34-46.

GuPTA, P.B. Y Gould, S.J. (1997), "Consumers' Perceptions of the ethics and acceptability of Product Placement in movies: Product Category an individual differences", Journal of Current Issues and Research in Advertising, 19(1), 37-50.

GuPTA, P.B. y Gould, S.J. (2007), "Recall of Products Placed as Prizes Versus Commercials in Game Shows", Journal of Current Issues and Research in Advertising, 29(1), pp. 43-54.

Hackley, C. y Tiwsakul, A. (2006), "Entertainment Marketing and Experiential Consumption", Journal of Marketing Communications, 12(1), 63-75.

Hackley, C., Tiwsakul, A. y Szmigin, I. (2005), "Explicit, non-integrated product placement in British television programmes", International Journal of Advertising, 24(1), 95-111.
Hall, M. (2004), "Broadcast Sponsorship: How does it work", Admap, (449), 19-22.

Hang, H. y Auty, S. (2008), "Video Games, Processing Fluency and Children: Exploring Product Placement in New Media". En The Proceedings of the Society for Consumer Psychology 2008 Winter Conference, Society for Consumer Psychology: New Orleans.

Heath, R.G. (2000), "Low involvement processing - a new model of brands and advertising", International Journal of Advertising, 19(3), 287-298.

Heeter, C. y Greenberg, B.S. (1985), "Profiling the Zappers", Journal of Advertising Research, 25(2), 15-20.

Hetsroni, A. y Asya, I. (2002), "A Comparison of values in infomercials and commercials", Corporate Communications: An International Journal, 7(1), 34-45.

Homer, P. M. (1990), "The mediating role of attitude toward the ad: some additional evidence", Journal of Marketing Research, 27(1), 78-86.

Ip Network (2007), Sectorial Survey Television.

JAFFE, J. (2005), Life after the 30 secondsSecond Spot, John Wiley and Sons: New Jersey.

LaFerle, C. y Edwards, E. M. (2006), "Product Placement: How Brand appear on Television", Journal of Advertising, 35(4).65-86.

LAMAS, C. (2004), "Saturación, concentración, multimedia: algunos condicionantes de la eficacia de las campañas". En Seminario $20^{\circ}$ AEDEMO de Televisión, Aedemo: Santiago de Compostela.

LARDINOIT, T. (1998), "Effet modérateur de limplication durable sur l'efficacité mémorielle de la conjonction des parrainages terrain et TV". En Proceedings of the 14th Congress of the French Marketing Association AFM, Association Française de Marketing: Bordeaux. 261-276.

LARDINOIT, T. (1999), "Interaction des parrainages terrain et TV: impact sur deux niveaux 
de mémorisation". En Proceedings of the 15th Congress of the French Marketing Association AFM, Association Française de Marketing: Strasbourg. 585-602.

LAW, S. y BRAUn, K.A. (2000), "I'll Have What She's Having: Gauging the Impact of Product Placement on Viewers", Psychology and Marketing, (17) 12, 1059-1075.

LEE, M. y FABER, R.J. (2007), "Effects of product placement in on-line games on brand memory: a perspective of the limited-capacity model of attention", Journal of Advertising, 36(4), 75-90.

Ley General de la Comunicación AudioviSUAL, 7/2010, de 31 de marzo, Boletín Oficial del Estado (BOE) de 1 de abril. España.

Li, C. (2010), "Primacy effect or recency effect? A long-term memory test of Super Bowl commercials", Journal of Consumer Behaviour, 9(1), 32-44.

LorD, K. R. Y Burnkrant, R. E. (1993), "Attention versus distraction: The interactive effect of program involvement and attentional devices on commercial processing", Journal of Advertising, 22(1), 47-60.

LOWREY, T. M. (2006), "The relation between script readability and commercial memorability", Journal of Advertising, 35(3), 7-15.

Lowrey, T. M., Shrum, L. J. y MCCARTY, J. A. (2005), "The Future of Television Advertising". En Allan J. Kimmel (Ed.), Marketing Communication: Emerging Trends and Developments, Oxford University Press: New York. 113-132.

Madden, T. A., Allen, C.T. y Twible, J. L (1998), "Attitude toward the ad: An assessment of diverse measurement indices under different processing "sets"”, Journal of Marketing Research, 23(3), 242-252.

MASTERSON, R. (2005), "The importance of creative match in television sponsorship", International Journal of Advertising, 24(4), 505-526.

Matthes, J., Schemer, C. Y Wirth, W. (2007), "More than meets the eye: Investigating the hidden impact of brand placements in televi- sion magazines", International Journal of Advertising, 26(4), 477-503.

MCDANIEL, S.R. (1999), "An investigation of match-up effects in sport sponsorship advertising", Psychology \& Marketing, 16(2), 163-184.

Mckenzie, S. B., Lutz, R. J. y Belch, G. E. (1986), "The role of attitude toward the ad as a mediator of advertising affectiveness: A test of competing explanations", Journal of Marketing Research, 23(2), 130-143.

Media Planning Group (2007), Eficacia publicitaria de las acciones publicitarias especiales en televisión.

Mediaedge (2007), Panorama de la Televisión en España 2006, Madrid: Mediaedge.

Meir, R., Arthur, D., Tobin, J. Y MassingHAM, C. (1997), "Professional rugby league in Australia: a case study in sponsor awareness", Cyber-Journal of Sport Marketing, $1(2), 83-91$.

Miles, P. (2009), "Product Placements. The Impact of Placement Type and Repetition on Attitude", Journal of Advertising, 38(3), 2132.

Moldovan, S. E. (1985), "Copy factors related to persuasion scores", Journal of Advertising Research, 24(6), 16-22.

Moore, J.N., Pickett, G.M. Y Grove, S.J. (1999), "The impact of a video screen and rotational- ignage systems on satisfaction and advertising recognition", Journal of Services Marketing, 13(6), 453-468.

Moorman, M., Neijens, P. y Smit, E. (2005), "The Effects of Program Responses on the Processing of Commercials Placed at Various Positions in the Program and the Block", Journal of Advertising Research, 45(1), 49-59.

Moorman, M., Neijens, P. y Smit, E. (2007), "The Effects of Program Involvement on Commercial Exposure and Recall in a Naturalistic Setting", Journal of Advertising, 36(1), 121-137.

Norris, C. E., Colman, A. M., y Aleixo, P. A. (2003), "Selective exposure to television 
programmes and advertising effectiveness", Applied Cognitive Psychology, 17, 593606.

OfCOM (2005), Extension of the Television Without Frontiers Directive. Final Report. Septiembre, disponible en: $h t t p: / / w w w . o f c o m$. org.uk/research/tv/twf/twfreport, 3/06/2006.

PARDO, I. (2008), "Acciones especiales en TV: no todo suma igual". En Seminario $24^{\circ}$ AEDEMO de Televisión, Aedemo: Granada.

Pervan S.J. y Martin, B.A.S. (2002), "Product placement in US and New Zealand television soap operas: an exploratory study", Journal of Marketing Communications, 8(2), 101113.

Peter, J. y Olson, J. (2005), Consumer Behaviour and Marketing Strategy, McGraw Hill: UK.

Phillips, D. M. y Stanton, J. L. (2004), “Agerelated differences in advertising: Recall and persuasion", Journal of Targeting, Measurement and Analysis for Marketing, 13(1), 720.

Pieters, R. y Bijmolt, T. (1997), “Consumer Memory for Television Advertising: A Field Study of Duration, Serial Position, and Competition Effects", Journal of Consumer Research: An Interdisciplinary Quarterly, 23(4), 362-372.

Redondo, I. (2006), "Product-Placement Planning: How Is the Industry Placing Brands in Relation to Moviegoer Consumption?", Journal of International Consumer Marketing, (18) 4, pp. 33-60.

ReINAREs LARA, R. (2010), Estudio de la eficacia cognoscitiva de las formas publicitarias no convencionales en televisión: una aplicación empírica en el mercado Español, Tesis Doctoral, Universidad Rey Juan Carlos: Madrid.

Reinares, P. y Reinares, E. (2003), Fundamentos básicos de la gestión publicitaria en televisión, Esic Editorial: Madrid.

REINARES, P. y ReINARES, E. (2007), "Analysis of the contents of the advertising saturation on television", Esic Market, (126), 23-94.
Reinares, P. Y Reinares, E. (2011), "The effectiveness on new television advertising formats: a real-time experiment in Spain, Marketing Review St. Gallen, 5, 56-61.

Reinares, P. y ReInAREs, R. (2008), "Gestión del patrocinio televisivo: una propuesta basada en su valoración por las audiencias". En Pindado, J. y Payne, G. (Coord.), Estableciendo puentes en una economía global, Esic Editorial: Madrid. 158.

Reinares, P. y ReINAREs, R. (2009), "Managing television sponsorship: a proposal based on its assessment by agents in the Spanish advertising system", Journal of Sponsorship, 2(4), 354-366.

Reinares, P., Reinares, R. y Reinares, E. (2007), "Valoración de la eficacia de las formas publicitarias no convencionales en la televisión: un estudio exploratorio". En AYALA, J.C. y GRUPo FEDRA Universidad de La Rioja (Eds.), Conocimiento, Innovación y Emprendedores. Camino al Futuro, Universidad de La Rioja: Logroño. 202.

RomaniuK, J. (2009), "The Efficacy of BrandExecution Tactics in TV Advertising, Brand Placements, and Internet Advertising", Journal of Advertising Research, 49(2), 143-150.

Royo Vela, M. (2005), "Emotional and Informational Content of Commercials: Visual and Auditory Circumplex Spaces, Product Information and their Effects on Audience Evaluation", Journal of Current Issues and Research in Advertising, 27(2), 13-38.

Rubinson, J. (2009), "Empirical Evidence of TV Advertising Effectiveness", Journal of Advertising Research, 49(2), 220-226.

Ruiz MafÉ, C. y SANz Blas, S. (2007), “Teleshopping adoption by Spanish consumers", Journal of Consumer Marketing, (24) 4, 242- 250.

Russell C. A. y Stern, B. B. (2006), “Consumers, Characters and Product, A Balance Model of Sitcom Product Placements Effect", Journal of Advertising, 35(1), 7-21.

Russell, C.A. (2002), "Investigating the Effectiveness of Product-Placements in Television 
Shows: The Role of Modality and Plot Connection Congruence on Brand Memory and Attitude", Journal of Consumer Research, 29(3), 306-318.

SANZ Blas, S. (2007), "Mensaje publicitario y comprador televisivo. Cómo lograr la efectividad de la comunicación y la satisfacción del telecomprador", Estudios sobre consumo, 80, 61-70.

Schmid, A. (2003), "Are New TV Advertising Formats More Effective?", Unpublished report of experimental research for IP Deutschland and RTL Television, disponible en: http://www.ipbelgium.be/upload/TV/ marketing/formats/effectivesplit/newtvads.pd f, 20/05/2008.

Sharp, B., Beal, V. y Collins, M. (2009), "Television: back to the future", Journal of Advertising Research, 49(2), 211-219.

Singh, S. K., Balasubramanian, K. y ChaKRABORTY, G. (2000), "A Comparative analysis of three communication formats: advertising, infomercial, and direct experience", Journal of Advertising, 29(4), 59-75.

Singh, S. N. y Cole, C. A. (1993), "The Effects of Length, Content, and Repetition on Television Commercial Effectiveness," Journal of Marketing Research, 30 (1), 91-104.

Smit, E., Van Reijmersdal, E. A. y Neijens, P. (2009), "Today's practice of brand placement and the industry behind it", International Journal of Advertising, 28(5), 761-782.

SMITH, R. (1993), "Integrating Information from Advertising and Trial: Processes and Effects on Consumer Response to Product Information", Journal of Marketing Research, 30(2), 204-219.

Sofres A. M. (2008), Anuario de audiencias de televisión 2007, Madrid: Sofres Audiencia de Medios.

Speck, P. S. y Elliott, M. T. (1997). "Predictors of advertising avoidance in print and roadcast media", Journal of Advertising, 26(3), 61-76.

Stanton, J. L. y Burke, J. (1998), “Comparative effectiveness of executional elements in
TV advertising: 15- versus 30-second commercials", Journal of Advertising Research, 38(6), 7-14.

Tiwsakul, R., Hackley, C. Y Szmigin, I. (2005), "Explicit, Non-integrated Product Placement in British Television Programmes: an exploratory study", International Journal of Advertising, 24(1), 95-111.

Tomkins, S.S. (1970), A Theory of Memory. In Cognition and Affect, Little Brown \& Co: Boston.

Torrano, J. y Flores, E. (2005), "Principales Factores Determinantes de la Actitud hacia el Emplazamiento del Producto". En Actas del VIII Forum Internacional sobre Las Ciencias, las Técnicas y el Arte Aplicadas al Marketing. Academia y Profesión: Universidad Complutense de Madrid. 605627.

Tse, A.C.B. Y LeE, R.P.W. (2001), "Zapping behaviour during commercial breaks", Journal of Advertising Research, 41(3), 25-29.

VAKRATSAS, D. (2005), “Advertising response models with managerial impact: an agenda for the future", Applied Stochastic Models in Business and Industry, 21(4/5), 351-361.

VAlKemburG, P. y BuiJzen, M. (2005), "Identifying determinants of young Children's Brand Awareness: Television, Parents, and Peers", Journal of Applied Developmental Psychology, 26(4), 456-468.

VAn Reijmersdal, E.A., Neijens, P.C. y Smit, E.G. (2007), "Effects of TV brand placement on brand image", Psychology \& Marketing, (24) 5, pp. 403-420.

Von Rimscha, M.B., Rademacher, P., ThoMAS, N. y SIEGERT, G. (2008), "The Future of TV Commercials: Not as Bleak as Proclaimed," Journal of Media Business Studies, 5(2), 23-46.

WALLiSER, B. (1994), "Les déterminants de la mémorisation des sponsors", Revue Française du Marketing, (150), pp. 83-95.

WeBB, P. H. y RAY, M. L. (1979), "Effects of TV clutter", Journal of Advertising Research, 19(3), 7-12. 
WILBUR, K. (2008), "How the digital video recorder changes traditional television advertising", Journal of Advertising, 37(1), 143-150.

Woods, L. (2008), "The consumer and advertising regulation in the Television Without Frontiers and Audiovisual Media Services
Directives", Journal of Consumer Policy, 31(1), 63-77.

ZinkHAN, G. M. y Fornell, C. R. (1985), “A test of two consumer response scales in advertising", Journal of Marketing Research, 20(2), 7-13.
Fecha recepción: 03/01/2011

Fecha aceptación: 01/09/2011 